\title{
Conductance of a spin-1 quantum dot: the two-stage Kondo effect
}

\author{
A. Posazhennikova, ${ }^{1, \oplus}$ B. Bayani, ${ }^{1}$ and P. Coleman ${ }^{2}$ \\ 1 Institut für Theoretische Festkörperphysik and DFG-Center of Functional Nanostructures, \\ Universität Karlsruhe, D-76128 Karlsruhe, Germany \\ ${ }^{2}$ Center for Materials Theory, Department of Physics and Astronomy, \\ Rutgers University, Piscataway, NJ 08854, USA
}

\begin{abstract}
We discuss the physics of a of a spin-1 quantum dot, coupled to two metallic leads and develop a simple model for the temperature dependence of its conductance. Such quantum dots are described by a two-channel Kondo model with asymmetric coupling constants and the spin screening of the dot by the leads is expected to proceed via a two-stage process. When the Kondo temperatures of each channel are widely separated, on cooling, the dot passes through a broad cross-over regime dominated by underscreened Kondo physics. A singular, or non-fermi liquid correction to the conductance develops in this regime. At the lowest temperatures, destructive interference between resonant scattering in both channels leads to the eventual suppression of the conductance of the dot. We develop a model to describe the growth, and ultimate suppression of the conductance in the two channel Kondo model as it is screened successively by its two channels. Our model is based upon large- $\mathrm{N}$ approximation in which the localized spin degrees of freedom are described using the Schwinger boson formalism.
\end{abstract}

PACS numbers:

\section{INTRODUCTION}

The Kondo effect arises from the resonant spin-flip scattering of electrons off localized magnetic impurities, which manifest themselves through the anomalous transport and thermodynamic properties of dilute magnetic alloys 1 . A modern context for the physics of local moments is found within quantum dots 2 in where the Kondo effect is manifested as a zero-bias anomaly of the differential conductance. The high degree of tunability in quantum dots allows for realization of a number of interesting variants of the Kondo effect, including both the "over-screened" and "under-screened" Kondo models. For example, the Kondo effect has been observed in spin-1 quantum dots containing an even number of electrons, by tuning the dot to a singlet-triplet degeneracy point by applying a magnetic field ${ }^{3,4}$.

The physics of a single spin $1 / 2$ magnetic impurity, with no orbital degrees of freedom is described by the canonical one-channel Kondo model, where the loan spin is fully screened by the conduction electrons at low temperatures, forming a paramagnetic ground-state with excitations described by Landau Fermi liquid theory. Nozières and Blandin pointed out $t^{-\underline{5}}$ that in more realistic magnetic ions the orbital structure of the local impurity electron needs to be taken into account $(l \neq 0)$, giving rise to a much richer class of spin-screening phenomena. For a spin $S$ with unquenched orbital angular momentum $l$, there are $n_{l}=2 l+1$ screening channels. Depending on whether $n_{l}$ is greater than, equal to, or smaller than the number $n_{e}=2 S$ of Hund's coupled electrons in the local moment, the spin is said to be overscreened $(2 l+1>2 S)$, fully screened $(2 l+1=2 S)$ or underscreened $(2 l+1<2 S)$. In both the underscreened and overscreened cases, the excitations of the ground-state are no longer described by a Fermi liquid, and a variety of exotic non-Fermi liquid (NFL) phenomena can develop, as summarized in Table I. Unfortunately, most transition metal ions are described by the fully screened case, where $2 S=2 l+1$, so the underscreened and overscreened Kondo effect is not readily observed in bulk materials. For this reason, quantum dots provide an important alternative milieu for the study of unconventional Kondo screening phenomena.

Most recent interest has focused on the case of the overscreened multichannel Kondo model. From detailed Bethe ansatz $z^{\underline{6}}$ and conformal field theory solutions ${ }^{\underline{7}}$ the low-energy physics of the overscreened $S=1 / 2$ Kondo effect is known to involve a non-trivial fixed point, with singular power-law behavior of the specific heat $C \sim T^{\frac{4}{n_{l}+2}}$ and magnetic susceptibility. (Logarithmic corrections to the specific heat appear in the two-channel case). A possible realization of this kind of behavior in the heavy electron compound $U B e_{13}$ has been proposed by Cox, where quadrupole fluctuations of singlet uranium ions may assume the role of spin fluctuations in the conventional Kondo effect $\frac{8,9}{9}$.

In point of fact, a spin- $1 / 2$ quantum dot coupled to two leads does not provide a realization of the two-channel Kondo effect, because the localized spin couples to a specific combination of the lead electrons forming a onechannel Kondo model ${ }^{10}$. A proposal to overcome this problem through the coupling of a quantum dot to a strongly interacting "quantum box" has been advanced by Oreg and Goldhaber-Gordon $\frac{11}{1}$, and the first, preliminary indications of the the two-channel Kondo effect have been recently reported ${ }^{12}$.

By contrast, realization of the underscreened Kondo effect requires a quantum dot with a higher spin $S>1 / 2$. In most quantum dots, electrons fill the dot in accordance with the Pauli principle, forming a singlet state when the number of electrons is even, and for the most part, zero- 
TABLE I: Ground state properties of the Kondo model with impurity spin $S$ and number of channels $n_{l}$. $C_{v}$ is the specific heat, and $\chi$ the magnetic susceptibility. In the over-screened Kondo effect, electron-scattering remains inelastic in the ground-state.

\begin{tabular}{|c|c|c|c|c|c|}
\hline Kondo effect & Examples & GS & Phase shift $\delta(\epsilon)$ & $C_{v}$ & $\chi$ \\
\hline Fully Screened & $\begin{array}{c}S=1 / 2 ; n_{l}=1 \\
S=1 ; n_{l}=2\end{array}$ & $\begin{array}{c}\text { Fermi } \\
\text { Liquid }\end{array}$ & $\frac{\pi}{2}+\alpha \epsilon$ & $C_{v} \sim T$ & $\sim \frac{1}{T_{K}}$ \\
\hline Under Screened & $S=1 ; n_{l}=1$ & $\begin{array}{c}\text { Singular } \\
\text { Fermi Liquid }\end{array}$ & $\frac{\pi}{2}+\frac{\alpha}{\ln \left(T_{K} / \epsilon\right)}$ & $\sim \frac{1}{T \ln ^{4}\left(T / T_{K}\right)}$ & $\sim \frac{1}{B \ln ^{2}\left(B / T_{K}\right)}$ \\
\hline Over Screened & $\begin{array}{c}S=1 / 2 ; n_{l}=2 \\
S=1 ; n_{l}=3\end{array}$ & \begin{tabular}{|c|} 
Non \\
Fermi Liquid
\end{tabular} & $\begin{array}{c}\text { inelastic } \\
\text { scattering }\end{array}$ & $\begin{array}{l}\sim T \ln T \\
\sim T^{4 / 5}\end{array}$ & $\begin{array}{c}\sim \ln T \\
\sim T^{-1 / 5}\end{array}$ \\
\hline
\end{tabular}

bias anomalies are observed in dots with odd numbers of electrons ${ }^{13,14}$. A sequence of more recent experiments have shown that zero-bias anomalies associated with a Kondo effect can also occur in quantum dots with even occupancies, where Hund's coupling between the electrons lead to novel degeneracies, either through the formation of higher spin states, or through the accidental degeneracy of singlet and triplet states. The observation of a zero-bias anomaly in even integer quantum dots was first reported by Schmid et al ${ }^{15}$ who tentatively identified the phenomenon with a triplet ground state of the quantum dot. Sasaki et $a^{\underline{3}}$ later discovered a zero-bias anomaly in even electron quantum dots that are tuned to the degeneracy point between singlet and triplet states. Most recently, Kogan et a ${ }^{\underline{4}}$ have shown that the singlet - triplet excitation energy in lateral quantum dots can be tuned by the gate voltage, explicitly demonstrating that the zero bias anomaly develops once the triplet state drops below the singlet configuration.

Pustilnik and Glazman ${ }^{16}$ have shown that a spin-1 quantum dot is associated with two screening channels which fully screen the local moment at the lowest temperatures. The two channels arise because the two electrons in a spin-1 dot occupy two separate orbitals which couple differently to the leads. At low temperatures the conductance of the Fermi liquid that emerges is governed by the interference between the two channels

$$
G=2 \frac{e^{2}}{h} \sin ^{2}\left(\delta_{1}-\delta_{2}\right)
$$

where $\delta_{1}$ and $\delta_{2}$ are the scattering phase shifts of the two screening channels ${ }^{16}$. According to this line of reasoning, the development of a unitary phase shift in each channel, $\delta_{1}=\delta_{2}=\pi / 2$ leads to a complete suppression of the zero bias anomaly in a triplet quantum $\operatorname{dot}^{17}$.

The two separate screening channels of a spin-1 quantum dot are associated with two different antiferromagnetic coupling constants $J_{\lambda}(\lambda=1,2)$. Since the Kondo temperature depends exponentially on the coupling constants, $T_{K \lambda}=D \sqrt{J_{\lambda} \rho} \exp \left(-\frac{1}{J_{\lambda} \rho}\right) \quad(\rho$ is the density of states and $D$ is the band width), a rather modest difference in coupling constants can produce Kondo temperatures separated by decades of temperature, $T_{K 1}>>$
$T_{K 2}$. Over the intervening temperature range, defined as $\ln \left(T_{K 1}\right) \gg \ln (T) \gg \ln \left(T_{K 2}\right)$, the physics of the dot is expected to be dominated by the underscreened fixed point, or one-channel spin-1 Kondo model. In the underscreened spin-1 Kondo, the residual spin-1/2 moment is ferromagnetically coupled to leads, with a coupling that scales asymptotically towards zero ${ }^{5}$. Recent studies of this problem have argued that the ground-state which develops is a "singular Fermi liquid", in which the electrons are elastically scattered with unitary phase shift, but in which the logarithmically decaying coupling to the partially screened local moment leads to a singular energy dependence in the scattering phase shift and a divergence in the resulting quasiparticle density of states $18,19,20$. This singular behavior, has a number of observable consequences for the conductance which if confirmed, will provide a first experimental realization of singular Fermi liquid behavior of an underscreened local moment.

In this paper we present an analysis of the transport properties of a spin-1 quantum dot in two cases: first for one screening channel and then for two active screening channels. The initial part of our paper provides a detailed treatment of the singular conductance properties previously predicted by us in a short paper on this topic $^{21}$. The second part of the paper extends this earlier work to take account of the second-channel screening processes and the interference it gives rise to. One of the main results of this new analysis, is the development for an approximate expression for the temperature dependence of the linear conductance. We find that the linear conductance can be divided into a coherent part, and an incoherent part,

$$
G=G_{c o h}+G_{i n c}
$$

where

$G_{c o h}=\frac{N e^{2}}{h}(2 \alpha \beta)^{2} \int d \omega\left(-\frac{d f(\omega)}{d \omega}\right)\left|\pi \rho\left(T_{1}(\omega)-T_{2}(\omega)\right)\right|^{2}$

defines the coherent conductance through the twochannel and

$$
G_{i n c}=\frac{N e^{2}}{h}(2 \alpha \beta)^{2} \int d \omega\left(-\frac{d f(\omega)}{d \omega}\right)
$$




$$
\times \pi \rho\left[\tau_{1}^{-1}(\omega)+\tau_{2}^{-1}(\omega)\right]
$$

the additional contribution due to incoherent transport, where " $T_{1}$ " and " $T_{2}$ " refer to the scattering t-matrices in the two screening channels of the quantum dot, $\alpha$ and $\beta$ define the relative amplitudes of channel 1 in the left and right channels respectively and

$$
\tau_{\lambda}^{-1}=\operatorname{Im} T_{\lambda}(\omega)-\pi \rho\left|T_{\lambda}(\omega)\right|^{2} . \quad(\lambda=1,2)
$$

are the inelastic scattering rates in each channel, defined by the deviation of the imaginary part of the t-matrix from the value expected from the optical theorem.

\section{CONSTRUCTION OF THE HAMILTONIAN}

Our initial model Hamiltonian for a spin-1 quantum dot coupled to two leads can be divided up into a Hamiltonian for the leads $\left(H_{L}\right)$, the quantum dot $\left(H_{D}\right)$ and the coupling between the leads and the $\operatorname{dot}\left(H_{C}\right)$ as follows

$$
H=H_{L}+H_{C}+H_{D}
$$

where

$$
H_{L}=\sum_{\substack{k \sigma \\ \gamma=L, R}} \epsilon_{k} c_{k \gamma \sigma}^{\dagger} c_{k \gamma \sigma}
$$

describes the electrons in the left and right leads,

$$
H_{C}=t_{1} \sum_{k}\left(\psi_{k \sigma}^{\dagger} d_{1 \sigma}+\text { H.c. }\right)+t_{2} \sum_{k}\left(\varphi_{k \sigma}^{\dagger} d_{2 \sigma}+\text { H.c. }\right) \text {, }
$$

describes the hybridization in two channels between the lead electrons and the quantum dot through the contacts. Here $d_{1 \sigma}^{\dagger}$ and $d_{2 \sigma}^{\dagger}$ create electrons in two orthogonal states of the quantum dot and

$$
\begin{aligned}
& \psi_{k \sigma}^{\dagger}=\alpha c_{k L \sigma}^{\dagger}+\beta c_{k R \sigma}^{\dagger} \\
& \varphi_{k \sigma}=-\beta c_{k L \sigma}^{\dagger}+\alpha c_{k R \sigma}^{\dagger},
\end{aligned}
$$

create the linear combinations of conduction electrons that hybridize with these two orthogonal scattering channels. The Hamiltonian of the spin-1 quantum dot can be written

$$
\begin{aligned}
H_{D} & =-E_{1} n_{d 1}-E_{2} n_{d 2}+U_{1} n_{d 1 \uparrow} n_{d 1 \downarrow}+U_{2} n_{d 2 \uparrow} n_{d 2 \downarrow} \\
& +U_{12} n_{d 1} n_{d 2}-J\left[\vec{S}_{1} \cdot \vec{S}_{2}\right] .
\end{aligned}
$$

Here $-E_{\lambda}(\lambda=1,2)$ are the energies of the two oneparticle states and $n_{d \lambda}=\sum_{\lambda} d_{\lambda \sigma}^{\dagger} d_{\lambda \sigma}(\lambda=1,2)$ are the occupancy of the two channels. $U_{1}, U_{2}$ and $U_{12}$ are the intra and inter-channel Coulomb interactions and lastly $\vec{S}_{\lambda}=\frac{1}{2} d_{\lambda \alpha}^{\dagger} \vec{\sigma}_{\alpha \beta} d_{\lambda \beta}$ are the spin operators for the two channels and $J$ is the (ferromagnetic) Hund's exchange coupling between the spins.

In general, we are interested in the case where the oneparticle energies $-E_{1}$ and $-E_{2}$ are negative, but $U_{1}, U_{2}$

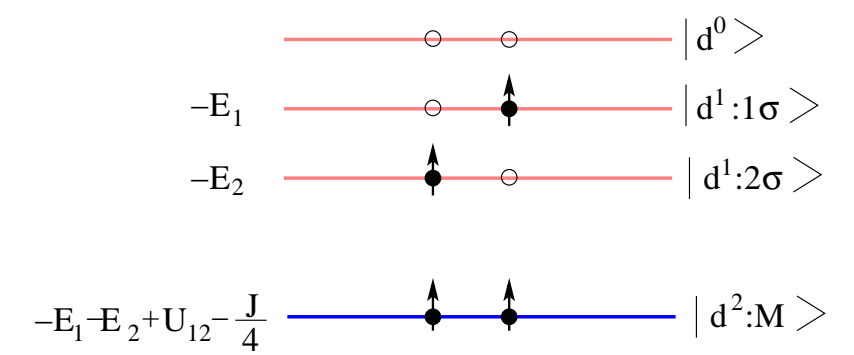

FIG. 1: Energy diagram of a spin-1 quantum dot with strong Hund's coupling.

are large quantities that restrict double occupancy of either d-state. Finally, the Hund's interaction $J$ is assumed to be large restricting the doubly occupied state to be a spin-1 triplet state. In this situation, the quantum dot is restricted to the states

$$
\begin{aligned}
\left|d^{0}\right\rangle & \equiv|0\rangle, \\
\left|d^{1}: 1 \sigma\right\rangle & =d_{1 \sigma}^{\dagger}\left|d^{0}\right\rangle, \quad\left|d^{1}: 2 \sigma\right\rangle=d_{2 \sigma}^{\dagger}\left|d^{0}\right\rangle, \\
\left|d^{2}: M\right\rangle & \equiv \begin{cases}d_{1 \uparrow}^{\dagger} d_{2 \uparrow}^{\dagger}\left|d^{0}\right\rangle, & (M=1) \\
\frac{1}{\sqrt{2}}\left(d_{1 \downarrow}^{\dagger} d_{2 \uparrow}^{\dagger}+d_{1 \uparrow}^{\dagger} d_{2 \downarrow}^{\dagger}\right)\left|d^{0}\right\rangle, & (M=0)(8) \\
d_{1 \downarrow}^{\dagger} d_{2 \downarrow}^{\dagger}\left|d^{0}\right\rangle, & (M=-1)\end{cases}
\end{aligned}
$$

The energy of the $d^{2}$ triplet states is $E_{1}+E_{2}-J / 4+U_{12}$ (see Fig.1).

We can project the Hamiltonian onto this Hilbert space by combining the d-electron operators with a generalized Gutzwiller projection operator as follows,

$$
d_{\lambda \sigma}^{\dagger} \rightarrow P_{T} d_{\lambda \sigma}^{\dagger} \equiv X_{\lambda \sigma}^{\dagger}
$$

where

$$
P_{T}=P_{G 1} P_{G 2}\left(1+\vec{S}_{1} \cdot \vec{S}_{2}-\frac{n_{d 1} n_{d 2}}{4}\right)
$$

and

$$
P_{G \lambda}=\left(1-n_{d \lambda \uparrow} n_{d \lambda \downarrow}\right), \quad(\lambda=1,2)
$$

is the Gutzwiller projector for channel one and two. Here, we have used Hubbard's "X" notation to describe the projected d-electron fields. With this notation, we can write our projected Hamiltonian as

$$
\begin{aligned}
H & =\sum_{k \sigma} \epsilon_{k} \psi_{k \sigma}^{+} \psi_{k \sigma}+\sum_{k \sigma} \epsilon_{k} \varphi_{k \sigma}^{+} \varphi_{k \sigma} \\
& +t_{1} \sum_{k}\left(\psi_{k \sigma}^{+} X_{1 \sigma}+h . c .\right)+t_{2} \sum_{k}\left(\varphi_{k \sigma}^{+} X_{2 \sigma}+\text { h.c. }\right) \\
& -E_{1} \sum_{\sigma} X_{1 \sigma}^{+} X_{1 \sigma}-E_{2} \sum_{\sigma} X_{2 \sigma}^{+} X_{2 \sigma} \\
& +\left(U_{12}-\frac{J}{4}\right) n_{d 1} n_{d 2}
\end{aligned}
$$

The main interactions in this Hamiltonian are hidden in the constraints. In the Kondo limit, where this Hamiltonian is dominated by excitations between the $d^{2}$ and $d^{1}$ 
state, the final interaction term has the effect of replacing the excitation energy

$$
-E_{\lambda} \rightarrow E\left(d^{2}\right)-E\left(d^{1}\right)=-E_{\lambda}-\frac{J}{4}+U_{12}
$$

With this observation, we shall eliminate the final interaction from the Hamiltonian, absorbing its effect into the above redefinition of $E_{\lambda}$. (Another way is to set $\left.U_{12}=J / 4\right)$.

In the extreme Kondo limit where the dot is predominantly in the $\left|d^{2}\right\rangle$ state, the $d^{2} \leftrightarrow d^{1}$ charge fluctuations can be eliminated from this Hamiltonian via a Schrieffer Wolff transformation to give

$$
\begin{aligned}
H=H_{L} & +J_{1} \sum_{k, k^{\prime} \alpha \beta} \psi_{k \alpha}^{\dagger} \vec{\sigma}_{\alpha \beta} \psi_{k^{\prime} \beta} \cdot \vec{S} \\
& +J_{2} \sum_{k, k^{\prime} \alpha \beta} \phi_{k \alpha}^{\dagger} \vec{\sigma}_{\alpha \beta} \phi_{k^{\prime} \beta} \cdot \vec{S}
\end{aligned}
$$

where

$$
J_{1}=\frac{\left(t_{1}\right)^{2}}{\frac{J}{4}+E_{1}-U_{12}}, \quad J_{2}=\frac{\left(t_{2}\right)^{2}}{\frac{J}{4}+E_{2}-U_{12}},
$$

are the two antiferromagnetic coupling constants, corresponding to two orthogonal channels.

\section{SCHWINGER BOSON REPRESENTATION}

A very convenient way to treat the Gutzwiller operators in this Hamiltonian is to use a combination of slave fermions and Schwinger bosons. If we describe the empty state $\left|d^{0}\right\rangle=\chi_{2}^{\dagger} \chi_{1}^{\dagger}|0\rangle$ as a pair of two slave fermions, then the singly occupied states are written

$$
\begin{aligned}
& \left|d^{1}: 1 \sigma\right\rangle \equiv b_{\sigma}^{\dagger} \chi_{1}\left|d^{0}\right\rangle=-b_{\sigma}^{\dagger} \chi_{2}^{\dagger}|0\rangle \\
& \left|d^{1}: 2 \sigma\right\rangle \equiv b_{\sigma}^{\dagger} \chi_{2}\left|d^{0}\right\rangle=b_{\sigma}^{\dagger} \chi_{1}^{\dagger}|0\rangle
\end{aligned}
$$

while the doubly occupied states are given by

$$
\left|d^{2}: M\right\rangle=\left\{\frac{1}{\sqrt{2}}\left(b_{\uparrow}^{\dagger}\right)^{2}|0\rangle, b_{\uparrow}^{\dagger} b_{\downarrow}^{\dagger}|0\rangle, \frac{1}{\sqrt{2}}\left(b_{\downarrow}^{\dagger}\right)^{2}|0\rangle,\right\}
$$

In this representation, the Hubbard operators are written

$$
X_{1 \sigma}^{\dagger}=\frac{1}{\sqrt{n_{b}}} b_{\sigma}^{\dagger} \chi_{1}, \quad X_{2 \sigma}^{\dagger}=\frac{1}{\sqrt{n_{b}}} b_{\sigma}^{\dagger} \chi_{2},
$$

The prefactors $\frac{1}{\sqrt{n_{b}}}\left(n_{b}\right.$ is the number of Schwinger bosons) are needed to guarantee the normalization of the wavefunction. Thus

$$
\left|d^{2}: M=1\right\rangle=X_{1 \uparrow}^{\dagger} X_{2 \uparrow}^{\dagger}|0\rangle=\frac{1}{\sqrt{2}}\left(b_{\uparrow}^{\dagger}\right)^{2}|0\rangle
$$

which is the right normalization. Similarly

$$
P_{T} d_{1 \uparrow}^{\dagger} d_{2 \downarrow}^{\dagger}\left|d^{0}\right\rangle=X_{1 \uparrow}^{\dagger} X_{2 \downarrow}^{\dagger}\left|d^{0}\right\rangle
$$

$$
=\frac{1}{\sqrt{2}} b_{\uparrow}^{\dagger} b_{\downarrow}^{\dagger}\left|d^{0}\right\rangle=\frac{1}{\sqrt{2}}\left|d^{2}: m=0\right\rangle .
$$

which consistently normalizes the matrix element

$$
\left\langle d^{2}: m=0\left|X_{1 \uparrow}^{\dagger}\right| d^{1}: 1 \downarrow\right\rangle=\frac{1}{\sqrt{2}} .
$$

The physical Hilbert space is defined by those states where

$$
n_{b}+n_{\chi 1}+n_{\chi 2}=2 S
$$

\section{A. Large-N expansion}

To develop a controlled many body treatment of (12) we shall employ a large- $N$ expansion, extending the number of spin components $\sigma$ from two to $N$. For the work in this paper, we are interested in the approach to the Kondo limit of this problem, where the amplitude of the valence fluctuations are small, and in this limit, we shall replace $\sqrt{n_{b}} \rightarrow \sqrt{2 S}$ in (16) the above expression. With this device,

$$
t_{\lambda} X_{\lambda \sigma}^{\dagger} \longrightarrow \frac{\tilde{t}_{\lambda}}{\sqrt{N}} b_{\sigma}^{\dagger} \chi_{\lambda}, \quad(\lambda=1,2)
$$

where $N$ is the spin degeneracy and $\tilde{t}_{\lambda}=t_{\lambda} / \sqrt{2 S / N}$. The model Hamiltonian is then

$$
\begin{aligned}
H & =\sum_{k \sigma} \epsilon_{k} \psi_{k \sigma}^{+} \psi_{k \sigma}+\sum_{k \sigma} \epsilon_{k} \varphi_{k \sigma}^{+} \varphi_{k \sigma} \\
& +\frac{\tilde{t}_{1}}{\sqrt{N}}\left(\psi_{k \sigma}^{+} \chi_{1}^{\dagger} b_{\sigma}+h . c .\right)+\frac{\tilde{t}_{2}}{\sqrt{N}} \sum_{k}\left(\varphi_{k \sigma}^{+} \chi_{2}^{\dagger} b_{\sigma}+\text { h.c. }\right) \\
& +E_{1} \chi_{1}^{\dagger} \chi_{1}+E_{2} \chi_{2}^{\dagger} \chi_{2} .
\end{aligned}
$$

where it is understood that as $N$ becomes large, $\tilde{t}_{1,2}$ is kept fixed. There is one additional trick required to preserve a finite scattering phase shift as $N \rightarrow \infty$. For this task, we introduce $K=k N$ bosonic "replicas", where $k$ is maintained fixed as $N \rightarrow \infty$. With this device, in strong coupling $K$ bosons bind into the Kondo singlet and we can produce a large $N$ mean field theory in which the scattering phase shift is $\delta=\pi k$, with the qualitatively correct logarithmic energy dependences 20 . The Hamiltonian used in the large $N$ expansion is then

$$
\begin{aligned}
H & =\sum_{k \sigma} \epsilon_{k} \psi_{k \sigma}^{+} \psi_{k \sigma}+\sum_{k \sigma} \epsilon_{k} \varphi_{k \sigma}^{+} \varphi_{k \sigma} \\
& +\frac{\tilde{t}_{1}}{\sqrt{N}} \sum_{k \sigma \mu}\left(\psi_{k \sigma}^{+} \chi_{1 \mu}^{\dagger} b_{\sigma \mu}+\text { h.c. }\right) \\
& +\frac{\tilde{t}_{2}}{\sqrt{N}} \sum_{k \sigma \mu}\left(\varphi_{k \sigma}^{+} \chi_{2 \mu}^{\dagger} b_{\sigma \mu}+h . c .\right) \\
& +\sum_{\mu}\left(E_{1} \chi_{1 \mu}^{\dagger} \chi_{1 \mu}+E_{2} \chi_{2 \mu}^{\dagger} \chi_{2 \mu}\right)
\end{aligned}
$$




$$
+\lambda\left(n_{b}+n_{\chi 1}+n_{\chi 2}-2 S K\right)
$$

where the sum over $\mu$ runs from 1 to $k N$, while the last term imposes the generalized constraint

$$
n_{b}+n_{\chi 1}+n_{\chi 2}=2 S K
$$

Notice how the large $N$ model Hamiltonian (22) is obtained by replacing

$$
t_{\lambda} \longrightarrow \frac{\tilde{t}_{\lambda}}{\sqrt{N}}, \quad X_{\lambda \sigma}^{\dagger} \rightarrow \sum_{\mu=1, K} b_{\sigma \mu}^{\dagger} \chi_{\lambda \mu} .
$$

in (12). Section IV examines in the underscreened onechannel regime, where $\tilde{t}_{2}=0$. Section $\nabla$ proceeds with a discussion of the full Hamiltonian.

\section{ONE-CHANNEL SPIN-1 QUANTUM DOT: UNDERSCREENED KONDO EFFECT}

\section{A. The singular Fermi liquid}

In this section we discuss the limit where the Kondo temperature of the second-channel $T_{K 2}$ is negligibly small, so that the physics of the quantum dot is governed by an underscreened Kondo effect. We present a large $N$ approximation to the physics of this underscreened limit.

The Hamiltonian that governs this behavior is derived from the Hamiltonian (22) with $\tilde{t}_{2}=0$ and $E_{2}=0$. Much is known about the equilibrium physics of this model. At low temperatures, the spin is partially screened from spin $S$ to spin $S-(1 / 2)$. The residual moment is ferromagnetically coupled to the conduction sea, with a residual coupling that slowly flows to weak coupling according to

$$
J \rho(\Lambda)=-\frac{1}{\ln \left(\frac{T_{K 1}}{\Lambda}\right)}+O\left(\frac{1}{\ln ^{2}\left(\frac{T_{K 1}}{\Lambda}\right)}\right)
$$

where $\Lambda \sim \max \left(T, \mu_{B} B\right)$ is the characteristic cut-off energy scale, provided in equilibrium, by the temperature or magnetic field. At low energies and temperatures, the partially screened magnetic moment scatters electrons elastically, with a unitary phase shift, however the coupling to the residual spin $\left(S-\frac{1}{2}\right)$ gives rise to a singular energy dependence of the scattering phase shift. The low energy scattering phase shift can be deduced from scaling theory to have the asymptotic form

$$
\delta(\omega)=\frac{\pi}{2} \pm \pi \rho J(\omega)=\frac{\pi}{2}\left(1 \pm \frac{\left(S-\frac{1}{2}\right)}{\ln \left(T_{K 1} / \omega\right)}\right) .
$$

for the "up" and "down" spin channels. The logarithmic term on the right hand side is produced by the residual coupling between the electrons and the partially screened moment. While the electrons at the Fermi energy scatter elastically off the local moment with unitary scattering phase shift, as in a Fermi liquid, the logarithmically singular dependence of the phase shift leads to a divergent density of states, $N(\omega) \sim \frac{1}{\pi} \frac{d \delta(\omega)}{d \omega} \sim \frac{1}{|\omega|}$, which means that we can not associate this state with a bona-fide Landau Fermi liquid. For this reason, the ground-state of the underscreened Kondo model has recently been called a "singular Fermi liquid" 19 .

These singular features of the underscreened Kondo effect are expected to manifest themselves in the properties of a triplet quantum dot, in the range where $T_{K 2}<<T<<T_{K 1}$. In this regime, the physics is dominated by the underscreened fixed point. For example, as a function of magnetic field, we expect the conductance to follow the simple relation

$$
G(B)=\frac{d I}{d V}=\frac{e^{2}}{h} \sin ^{2} \delta(B)
$$

so at low fields $T_{K 1}<<\mu_{B} B<<T_{K 2}$, the differential conductance will have the form

$$
G \sim \frac{e^{2}}{h}\left(1-\frac{\pi^{2}}{16} \frac{1}{\ln ^{2}\left(\frac{T_{K 1}}{\mu_{B} B}\right)}\right)
$$

Notice that the field derivative of the conductance diverges as $1 / B$

$$
\frac{d G}{d B}=\frac{1}{B} \frac{e^{2}}{h}\left(\frac{\pi^{2}}{8 \ln ^{3}\left[\frac{T_{K 1}}{\mu_{B} B}\right]}\right)
$$

at low fields. The prediction of the finite temperature, and finite voltage conductance can not be made exactly, however we expect the above form to hold, for the differential conductance at finite temperature or voltage, with an appropriate replacement of cut-offs, namely

$$
G(V, T) \sim \frac{e^{2}}{h}\left(1-\frac{\pi^{2}}{16} \frac{1}{\ln ^{2}\left(\frac{T_{K 1}}{\max (T, e V)}\right)}\right)
$$

so that over the temperature range dominated by the underscreened Kondo fixed point, the conductance will be a monotonically increasing function of decreasing temperature and voltage. Over this range, $d G / d T \sim 1 / T$ and $d G / d V \sim 1 / V$ will be divergent functions of temperature and voltage respectively.

\section{B. Dyson equations and one-channel t-matrix}

We now analyze the low energy behavior of the tmatrix in the large-N approach. Our development begins with a derivation of the Dyson equations for the Green's function of the conduction $\psi_{k \sigma}$-fermions $(G)$ that are coupled to the dot and the Green's function of auxiliary $\chi$-holons $(J)$ that describe the partial screening. In this large-N approach, we will show that the Green's function of the Schwinger bosons $B$ remains unrenormalized. 
In general, we will be dealing with a voltage-biased quantum dot. The Hamiltonian for the leads is given by

$$
H_{0}=\sum_{k \gamma \sigma}\left(\epsilon_{k}-\mu_{\gamma}\right) c_{k \gamma \sigma}^{\dagger} c_{k \gamma \sigma}, \quad(\gamma=R, L)
$$

where $\mu_{L}=+\frac{e V}{2}$ and $\mu_{R}=-\frac{e V}{2}$. The Green's function of the conduction electrons is given by

$$
g_{\gamma}\left(t-t^{\prime}\right)=-i \sum_{k}\left\langle T_{c_{K}} c_{k \gamma \sigma}(t) c_{k \gamma \sigma}^{\dagger}\left(t^{\prime}\right)\right\rangle
$$

where $c_{K}$ is the Keldysh contour. Following the standard procedure, we write this Green's function using the Larkin-Ovchinnikov representation, in terms of the advanced $\left(g^{A}\right)$, retarded $\left(g^{R}\right)$ and Keldysh Green's functions $g^{K}$ as follows

$$
\hat{g}_{\gamma}=\left[\begin{array}{cc}
g_{\gamma}^{R} & g_{\gamma}^{K} \\
0 & g_{\gamma}^{A}
\end{array}\right]=\left[\begin{array}{cc}
-i \pi \rho & -i 2 \pi \rho\left(1-2 f_{\gamma}\right) \\
0 & i \pi \rho
\end{array}\right]
$$

where $\rho$ is the electron density of states and

$$
f_{\gamma}(\epsilon) \equiv f\left(\epsilon-\mu_{\gamma}\right), \quad(\gamma=R, L)
$$

describes the Fermi function in the left and right-hand leads. The complete Green's function describing the two leads is then a four-dimensional, block-diagonal matrix

$$
\hat{g}_{\text {lead }}=\left(\begin{array}{cc}
\hat{g}_{L} & 0 \\
0 & \hat{g}_{R}
\end{array}\right)
$$

To obtain the corresponding Green's function in the channel basis, we carry out a unitary transformation, writing

$$
\hat{g}_{c h}=\left[\begin{array}{cc}
\hat{g}_{\psi \psi} & \hat{g}_{\psi \varphi} \\
\hat{g}_{\varphi \psi} & \hat{g}_{\varphi \varphi}
\end{array}\right]=R \hat{g}_{l e a d} R^{-1}
$$

where

$$
R=\left(\begin{array}{cc}
\alpha & \beta \\
-\beta & \alpha
\end{array}\right)
$$

transforms between the two bases. The block-diagonal components of the matrix $\hat{g}_{c h}$ are now

$$
\hat{g}_{\psi \psi}=\left[\begin{array}{cc}
-i \pi \rho & -2 i \pi \rho\left(1-2 f_{\psi}^{(0)}\right) \\
0 & i \pi \rho
\end{array}\right]
$$

and

$$
\hat{g}_{\varphi \varphi}=\left[\begin{array}{cc}
-i \pi \rho & -2 i \pi \rho\left(1-2 f_{\varphi}^{(0)}\right) \\
0 & i \pi \rho
\end{array}\right]
$$

where

$$
f_{\psi}^{(0)}(\omega)=\alpha^{2} f_{L}(\omega)+\beta^{2} f_{R}(\omega)
$$

and

$$
f_{\varphi}^{(0)}(\omega)=\beta^{2} f_{L}(\omega)+\alpha^{2} f_{R}(\omega)
$$

define weighted-averages of the distribution functions in the two leads. The subscripts (0) are included, to delineate these functions from the renormalized local conduction electron distribution functions that can, in principle develop when the leads are coupled.

There are no retarded or advanced components to the off-diagonal block matrices $\hat{g}_{c h}$, but the inter-channel Keldysh Green's function does become finite when there is a voltage between the two leads,

$$
\hat{g}_{\varphi \psi}(\omega)=\hat{g}_{\psi \varphi}(\omega)=\left[\begin{array}{cc}
0 & g_{\varphi \psi}^{K} \\
0 & 0
\end{array}\right]
$$

where

$$
g_{\psi \varphi}^{K}(\omega)=g_{\varphi \psi}^{K}(\omega)=(4 i \pi \rho) \alpha \beta\left(f_{R}(\omega)-f_{L}(\omega)\right),
$$

and in this way, a finite voltage mixes the two channels.

We shall return to these general expressions later. However, for the one-channel problem, only the $\psi$ electrons couple to the quantum dot, and so our interest now focuses on $g_{\psi \psi}$.

The Dyson equations for the propagators of conduction $\psi$-electrons and slave $\chi$-fermions (Fig.2) are

$$
\begin{aligned}
\hat{G}^{-1} & =\hat{g}_{\psi \psi}^{-1}-\hat{\Sigma}, \\
\hat{J}^{-1} & =\hat{J}_{0}^{-1}-\hat{\Pi},
\end{aligned}
$$

where

$$
\hat{g}_{\psi \psi}^{-1}=\left[\begin{array}{cc}
\frac{1}{-i \pi \rho} & \frac{2}{-i \pi \rho}\left(1-2 f_{\psi}\right) \\
0 & \frac{1}{i \pi \rho}
\end{array}\right]
$$

is the bare inverse conduction propagator and

$$
\hat{J}_{0}^{-1}=\left(\begin{array}{cc}
\left(\omega-E_{d}-\lambda+i \delta\right) & 0 \\
0 & \left(\omega-E_{d}-\lambda-i \delta\right)
\end{array}\right),
$$

is the inverse propagator of the $\chi$ fermion. Notice how the Keldysh component of $g_{\psi \psi}^{-1}=\frac{2}{-i \pi \rho}\left(1-2 f_{\psi}^{(0)}(\omega)\right)$ is finite, whereas the Keldysh component of $J_{0}^{-1}$ is infinitesimal, and has been dropped from the above equations.

The self-energies are generated in our large- $\mathrm{N}$ meanfield theory within the non-crossing approximation (NCA). The diagrammatic Dyson equations are shown on Fig.1. The boson is our large-N approach is approximated as a sharp excitation, with an average occupancy $\left\langle n_{b \sigma \mu}\right\rangle \equiv n_{B}=(2 S K) /(N K)$ (see Appendix I). In all our calculations, we make use of the bare Green's function for the Schwinger bosons

$$
\hat{B}_{0}=\left(\begin{array}{cc}
B_{0}^{R} & B_{0}^{K} \\
0 & B_{0}^{A}
\end{array}\right)
$$




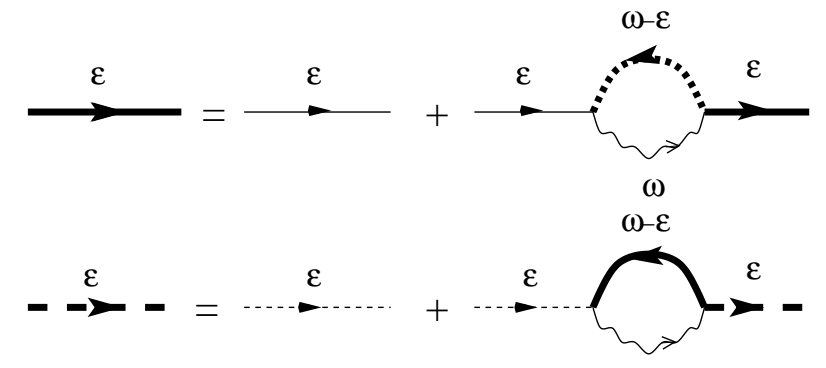

$\omega$

FIG. 2: The non-crossing approximation for the self-energies of conduction electrons and $\chi$ fermions. The solid line denotes the Larkin Ovchinnikov matrix propagator for the conduction electrons. The dashed line denotes the corresponding Green's function of the auxiliary $(\chi)$ fermions and the wavy line is the bosonic propagator. Thin lines denote the bare propagator and full lines the dressed propagator. Each vertex corresponds to the factor $i \frac{\tilde{t}}{\sqrt{N}}$.

where

$$
\begin{aligned}
B_{0}^{R, A} & =\frac{1}{\nu-\lambda \pm i \delta} \\
B_{0}^{K} & =\left(B_{0}^{R}-B_{0}^{A}\right) h_{B},
\end{aligned}
$$

where $h_{B}=1+2 n_{B}(\lambda)$.

¿From the Dyson equations we obtain sets of selfconsistent integral equations for the retarded, advanced and Keldysh self-energies (Appendix I). The self-energies for the slave $(\chi)$ fermion are

$$
\begin{aligned}
\Pi^{R}(\epsilon) & =-t^{2} n_{B}(\lambda) G^{A}(\lambda-\epsilon) \\
& -t^{2} \int_{-\infty}^{\infty} \frac{d \omega}{\pi} f_{\psi}(\omega) \frac{1}{\omega-\lambda+\epsilon+i \delta} \operatorname{Im}^{R}(\omega) \\
\Pi^{K}(\epsilon) & =-2 i t^{2} \operatorname{Im} G^{R}(\lambda-\epsilon)\left[n_{B}(\lambda)-f_{\psi}(\lambda-\epsilon)\right. \\
& \left.-2 n_{B}(\lambda) f_{\psi}(\lambda-\epsilon)\right] .
\end{aligned}
$$

The advanced self-energy is determined from the complex conjugate of the retarded self-energy $\Pi^{A}(\omega)=\Pi^{R}(\omega)^{*}$. In obtaining these results, we have assumed that the conduction electron Keldysh Green's function is determined by the relation

$$
G^{K}(\omega)=\left(G^{R}(\omega)-G^{A}(\omega)\right) h_{\psi}(\omega)
$$

where à priori, $h_{\psi}(\omega)=1-2 f_{\psi}(\omega)$ no longer equal to its equilibrium distribution.

The ratio of the Keldysh to the retarded self-energies self-consistently determines the $\chi$ fermion distribution functions

$$
h_{\chi} \equiv 1-2 f_{\chi}=\frac{\Pi^{K}}{\Pi^{R}-\Pi^{A}}=\frac{h_{\psi} h_{B}-1}{h_{B}-h_{\psi}} .
$$

Rearranging this expression gives

$$
h_{\psi}(\omega)=\frac{h_{\chi} h_{B}-1}{h_{B}-h_{\chi}} .
$$

The self-energies for the conduction electrons are

$$
\begin{aligned}
\Sigma^{R}(\epsilon) & =-t^{2} \frac{K}{N} n_{B}(\lambda) J^{A}(\lambda-\epsilon) \\
& -t^{2} \frac{K}{N} \int_{-\infty}^{\infty} \frac{d \omega}{\pi} f_{\chi}(\omega) \frac{1}{\omega-\lambda+\epsilon+i \delta} \operatorname{Im}^{R}(\omega) \\
\Sigma^{K}(\epsilon) & =-2 i t^{2} \frac{K}{N} \operatorname{Im}^{R}(\lambda-\epsilon)\left[n_{B}(\lambda)-f_{\chi}(\lambda-\epsilon)\right. \\
& \left.-2 n_{B}(\lambda) f_{\chi}(\lambda-\epsilon)\right] .
\end{aligned}
$$

and again, $\Sigma^{A}(\omega)=\Sigma^{R}(\omega)^{*}$. The ratio of Keldysh to the retarded/advanced Green's function is given by

$$
h_{\psi}(\omega)=\frac{\Sigma^{K}}{\Sigma_{\chi}^{R}-\Sigma_{\chi}^{A}}=\frac{h_{\chi} h_{B}-1}{h_{B}-h_{\chi}} .
$$

but this recovers exactly the result obtained in (39), showing that detailed balance is satisfied. However, we can't choose any distribution function $h_{\psi}$. If we go back to the original Dyson equation for the conduction electron Green's function (31), the Keldysh component of the Green's function is given by

$$
G^{K}=G^{R}\left[\Sigma^{K}-\left(g_{\psi \psi}^{-1}\right)^{K}\right] G^{A}
$$

But the distribution function associated with the selfenergy is determined by $\Sigma^{K}=h_{\psi}\left(\Sigma^{R}-\Sigma^{A}\right)$ whereas the distribution function associated with $\left[g_{\psi \psi}^{-1}\right]^{K}=$ $2 /(-i \pi \rho)\left(1-2 f_{\psi}^{(0)}\right)$ is the bare distribution function $h_{\psi}^{(0)}=1-2 f_{\psi}^{(0)}$. In this way, we see that our original assumption $G^{K}=\left(G^{R}-G^{A}\right) h_{\psi}$ requires that $h_{\psi}=h_{\psi}^{(0)}$. In other words, in large $N$ limit of the single-channel quantum dot electron distribution function is unaffected by the coupling to the dot.

We can summarize the results of our calculation of the Keldysh self-energies by providing the distribution functions that they generate. The distribution function of the auxiliary fermion is simply determined by the relationship

$$
f_{\chi}(\omega)=\frac{n_{b}\left[1-f_{\psi}(\omega)\right]}{n_{b}+f_{\psi}(\omega)}
$$

where $n_{b}=1 /\left(e^{\beta \lambda}+1\right)$ determines $\lambda$. This relationship can be simply understood as the result of detailed balance between rate of the decay processes $c \rightarrow b+\chi$ and $b+$ $\chi \rightarrow c$, and it reverts to the equilibrium Fermi Dirac distribution in the limit $V \rightarrow 0$.

We have solved the equations (37) and (40) numerically to obtain the energy-dependent $t$-matrix, given by

$$
\hat{t}_{\psi}(\omega)=\hat{\Sigma}(\omega)\left(1-\hat{g}_{\psi \psi}(\omega) \Sigma(\omega)\right)^{-1} .
$$

Actually, the Keldysh part of this quantity is determined simply from the relation $t_{\psi}^{K}=\left(t_{\psi}^{R}-t_{\psi}^{A}\right) h_{\psi}^{(0)}$, and the advanced part of the electron t-matrix can be simply written

$$
t_{\psi}^{A}(\omega)=\frac{\Sigma^{A}(\omega)}{\left(1-i \pi \rho \Sigma^{A}(\omega)\right)}
$$




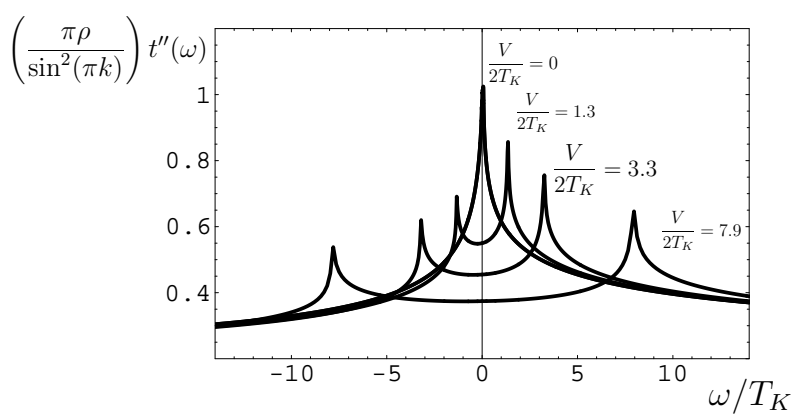

FIG. 3: Imaginary part of the t-matrix for a variety of voltages for $K / N=0.4$. As the voltage is increased, the singular central peak splits into two components.

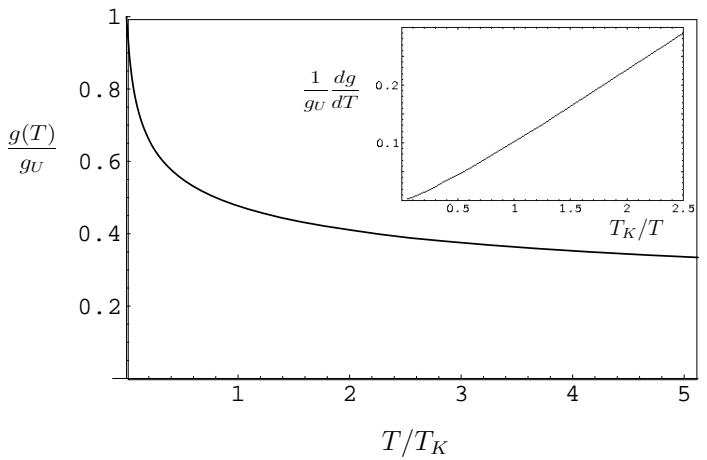

FIG. 4: Temperature dependence of the differential conductance, normalized with respect to $g_{U}=N \frac{e^{2}}{h} \sin ^{2}(\pi K / N)$ for the representative case $K / N=0.4$. Insert shows the $1 / T$ divergence of the derivative $d g / d T$.

The imaginary part of $t^{A}(\omega)$ determines the electronic density of states of the resonantly screened d-electrons. The voltage dependence of this quantity (at $T=0$ ) is shown in Fig.3. At zero voltage, the t-matrix contains a logarithmic divergence, which splits into two peaks at a finite voltage. In this large $N$ limit, the split Kondo resonance retains its singular structure. This is an artifact of taking a large $N$ limit in which the Schwinger bosons are preserve their sharp spectral structure.

\section{Conductance}

The conductance $G=\partial I / \partial V$ is defined by the temperature and voltage dependent current $^{22}$

$$
I(V, T)=\frac{e^{2}}{2 \hbar} N \rho \int \frac{d \omega}{\pi}\left[f_{L}(\omega)-f_{R}(\omega)\right] \operatorname{Im} t^{A}(\omega)
$$

where the advanced t-matrix is given by (44).

In Fig.4, we show the computed temperature dependent conductance. The temperature-dependent deviations from unitary conductance are determined

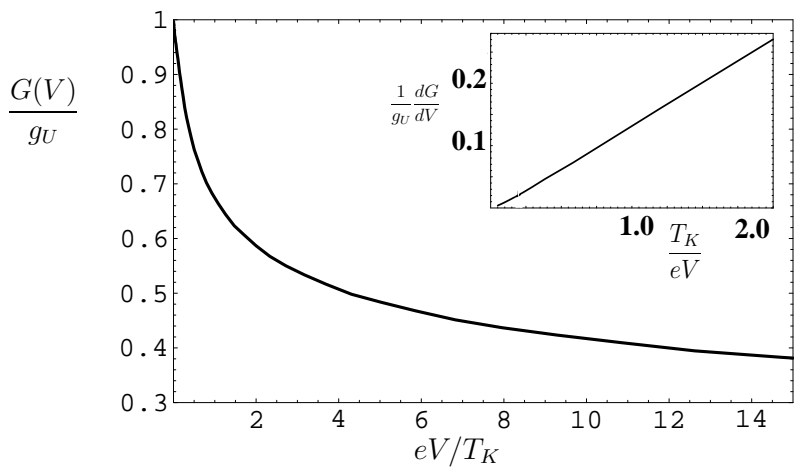

FIG. 5: Voltage-dependent conductance $G(V)=I(V) / V$ for the case $K / N=0.4$. Insert: derivative of $G(V)$ showing $1 / V$ divergence.

by the logarithmic singularity in the phase shift, and in our calculation, these are proportional to $1 / \ln \left(T_{K} / \max (e V, T)\right)$. There is a technical point here that needs some discussion. In the Schwinger boson approach, the number of bound bosons in the Kondo singlet does not exceed $N / 2$, which has the largest bound-state energy, so the region $K \geq N / 2$, does not propertly describe an underscreened Kondo model. Consequently, we are limited to static phase shifts $\delta=\pi(K / N)<\pi / 2$, so the particle-hole symmetric case $\delta=\pi / 2$ lies beyond the reach of this approach. This will leads to some important differences between the results of the current calculation and those expected in experiment. In general our solution does indeed capture the singular low energy behavior of the phase shift

$$
\delta_{c} \sim \pi k-\frac{\mathrm{cons}}{\ln \left[\frac{T_{K 1}}{T}\right]}
$$

Now the conductance $G$ depends on $\sin ^{2} \delta(V, T)$, so that for $k \neq 1 / 2$

$$
G \sim \sin ^{2} \delta=\sin ^{2}(\pi k)-\frac{\text { cons }}{\ln \left[\frac{T_{K 1}}{T}\right]}
$$

which is the form that our solutions follow. However, in the special case $k=1 / 2$, the coefficient of leading log in conductance vanishes, so the conductance involves square of the logarithm

$$
G \sim \sin ^{2}(\pi / 2)-\frac{\text { cons }}{\ln ^{2}\left[\frac{T_{K 1}}{T}\right]}
$$

In both cases, the temperature derivative of the conductance has the singular divergence $d G / d T \sim 1 / T$, a feature which can be tested experimentally.

Finally, Fig. 5 shows the voltage dependence of the conductance, which has a similar logarithmic singularity at low voltage. The insert shows $d^{2} I / d V^{2}$ versus $1 / \mathrm{eV}$. 


\section{TWO-CHANNEL SPIN-1 QUANTUM DOT: TWO-STAGE KONDO EFFECT}

We start with a derivation of the general current expression for the case of a two-channel quantum dot. In this section we consider the system, described by the Hamiltonian (21) and finite voltage. We show that at finite voltage the current has three contributions: contribution from each channel and the interference term.

\section{A. Derivation of the two-channel current expression}

The current from the dot into the left, and right hand leads is defined from

$$
I_{L, R}=-e\left\langle\dot{N}_{L, R}\right\rangle=-\frac{i e}{\hbar}\left\langle\left[H, N_{L, R}\right]\right\rangle .
$$

The non-zero contribution to the current derives from the commutator with the hybridization $H_{C}$ in equation (5). In equilibrium $I_{R}=-I_{L}=I$. The current into the right-hand lead is given by

$$
\begin{aligned}
I_{R} & =-\frac{i e}{\hbar} \sum_{k \sigma}\left[\beta t_{1}\left\langle X_{1 \sigma}^{\dagger} c_{k R \sigma}\right\rangle+\alpha t_{2}\left\langle X_{2 \sigma}^{\dagger} c_{k R \sigma}\right\rangle-\text { H.c. }\right] \\
& =-\frac{i e}{\hbar} \sum_{k \sigma} t_{1}\left[\alpha \beta\left(\left\langle X_{1 \sigma}^{\dagger} \varphi_{k \sigma}\right\rangle-\text { H.c. }\right)+\beta^{2}\left(\left\langle X_{1 \sigma}^{\dagger} \psi_{k \sigma}\right\rangle-\text { H.c. }\right)\right] \\
& -\frac{i e}{\hbar} \sum_{k \sigma} t_{2}\left[\alpha \beta\left(\left\langle X_{2 \sigma}^{\dagger} \psi_{k \sigma}\right\rangle-\text { H.c. }\right)+\alpha^{2}\left(\left\langle X_{2 \sigma}^{\dagger} \varphi_{k \sigma}\right\rangle-\text { H.c. }\right)\right] .
\end{aligned}
$$

Now the expectation of the occupancy of the dot in each channel is a constant in the steady state, so that

$$
\frac{d}{d t}\left\langle n_{d \lambda}\right\rangle=\frac{i}{\hbar}\left\langle\left[H_{C}, n_{d \lambda}\right]\right\rangle=0, \quad(\lambda=1,2)
$$

Carrying out the commutator, we obtain

$$
0=\frac{i}{\hbar} \sum_{k \sigma}\left[\left\langle X_{1 \sigma}^{\dagger} \psi_{k \sigma}\right\rangle-\text { H.c }\right]=-\frac{i}{\hbar} \sum_{k \sigma}\left[\left\langle X_{2 \sigma}^{\dagger} \varphi_{k \sigma}\right\rangle-\text { H.c }\right]
$$

From this consideration, we see that the second terms in equation (49) identically vanish, so that the current

$$
I=I_{R}=\frac{i e}{\hbar} \alpha \beta \sum_{k \sigma}\left[t_{1}\left(\left\langle X_{1 \sigma}^{\dagger} \varphi_{k \sigma}\right\rangle+\text { H.c. }\right)+t_{2}\left(\left\langle X_{2 \sigma}^{\dagger} \psi_{k \sigma}\right\rangle+\text { H.c. }\right]\right]
$$

A similar procedure for $J_{L}$ confirms that $J_{L}=-I$. Note, that in contrast to the Hamiltonian, the current is determined by the overlaps between the $\psi$-field with the $d_{2}$-electron and the $\varphi$-field with the $d_{1}$-electron on the dot.

The expectation values that enter into current are directly related to the equal-time Keldysh Green functions,

$$
\sum_{k \sigma}\left\langle\varphi_{k \sigma}^{\dagger}(t) X_{1 \sigma}(t)\right\rangle=\frac{N}{2 i} \int \frac{d \omega}{2 \pi} G_{1 \varphi}^{K}(\omega)
$$

and

$$
\sum_{k \sigma}\left\langle\psi_{k \sigma}^{\dagger}(t) X_{2 \sigma}(t)\right\rangle=\frac{N}{2 i} \int \frac{d \omega}{2 \pi} G_{2 \psi}^{K}(\omega),
$$

enabling us to write the current in the form

$$
\begin{array}{r}
I=\frac{N e}{2 \hbar} \alpha \beta \int \frac{d \omega}{2 \pi}\left[t_{1}\left(G_{\varphi 1}^{K}(\omega)-G_{1 \varphi}^{K}(\omega)\right)\right. \\
\left.+t_{2}\left(G_{\psi 2}^{K}(\omega)-G_{2 \psi}^{K}(\omega)\right)\right] .
\end{array}
$$

Combining the Dyson equations for the Green's functions $G_{1 \varphi}$ and $G_{2 \psi}$ with the expressions for bare Green's functions from Section IVB (for details see Appendix II) we obtain the general current expression in case of two channels

$$
I=N\left(I_{\varphi}+I_{\psi}+I_{\text {int }}\right),
$$

where

$$
\begin{gathered}
I_{\psi}=\frac{2 e}{\hbar}(\alpha \beta)^{2} t_{1}^{2} \rho \int d \omega\left(f_{L}(\omega)-f_{R}(\omega)\right) \operatorname{Im} D_{11}^{A} \\
I_{\varphi}=\frac{2 e}{\hbar}(\alpha \beta)^{2} t_{2}^{2} \rho \int d \omega\left(f_{L}(\omega)-f_{R}(\omega)\right) I m D_{22}^{A},
\end{gathered}
$$

where $D_{i j}\left(t-t^{\prime}\right)=\left\langle T_{c K} X_{i \sigma}(t) X_{j \sigma}^{\dagger}\left(t^{\prime}\right)\right\rangle$ is the Green's function of the electrons on the dot. 
The interference between the channels is induced by the terms

$$
\begin{array}{r}
I_{\text {int }}=-\frac{e}{2 \hbar} \alpha \beta t_{1} t_{2} i \rho \int d \omega\left\{D_{21}^{K}+D_{12}^{K}\right. \\
+\left(1-2 \beta^{2} f_{L}(\omega)-2 \alpha^{2} f_{R}(\omega)\right)\left(D_{21}^{A}-D_{12}^{R}\right) \\
\left.+\left(1-2 \alpha^{2} f_{L}(\omega)-2 \beta^{2} f_{R}(\omega)\right)\left(D_{12}^{A}-D_{21}^{R}\right)\right\}
\end{array}
$$

Notice that in the one-channel limit, $\left(t_{2}=0\right)$, the total current reduces to

$$
I=N I_{\psi}=\frac{N e}{\hbar} \int d \omega\left[f_{L}(\omega)-f_{R}(\omega)\right] \frac{\Gamma}{\pi} \operatorname{Im} D_{11}^{A}(\omega)
$$

recovering the Meir-Wingreen 22 expression for the current in a one channel quantum dot, with $\Gamma=$ $\Gamma_{L} \Gamma_{R} /\left(\Gamma_{L}+\Gamma_{R}\right)$, where $\Gamma_{L}=2 \pi \rho \alpha^{2} t_{1}^{2}$ and $\Gamma_{R}=$ $2 \pi \rho \beta^{2} t_{1}^{2}$.

\section{B. Approximate expression for the conductance}

In order to calculate the conductance, we need the Dyson equations for the Green's functions, defined by Hamiltonian (22). The Green's functions are defined in the same way as for the one-channel case and the Dyson equations (Fig 6) are

$$
\begin{gathered}
\left(\begin{array}{cc}
\hat{G}_{\psi \psi} & \hat{G}_{\psi \varphi} \\
\hat{G}_{\varphi \psi} & \hat{G}_{\varphi \varphi}
\end{array}\right)^{-1}=\left(\begin{array}{ll}
\hat{g}_{\psi \psi} & \hat{g}_{\psi \varphi} \\
\hat{g}_{\varphi \psi} & \hat{g}_{\varphi \varphi}
\end{array}\right)^{-1}-\left(\begin{array}{cc}
\hat{\Sigma}_{\psi \psi} & \hat{\Sigma}_{\psi \varphi} \\
\hat{\Sigma}_{\varphi \psi} & \hat{\Sigma}_{\varphi \varphi}
\end{array}\right) \\
\left(\begin{array}{ll}
\hat{J}_{11} & \hat{J}_{12} \\
\hat{J}_{21} & \hat{J}_{22}
\end{array}\right)^{-1}=\left(\begin{array}{cc}
\left(\hat{J}_{0}^{-1}\right)_{11} & 0 \\
0 & \left.\hat{(} \hat{J}_{0}^{-1}\right)_{11}
\end{array}\right)-\left(\begin{array}{ll}
\hat{\Pi}_{11} & \hat{\Pi}_{12} \\
\hat{\Pi}_{21} & \hat{\Pi}_{22}
\end{array}\right)
\end{gathered}
$$

Here

$$
\begin{aligned}
\left(J_{0}^{-1}\right)_{i i}^{R, A} & =\omega-E_{i}-\lambda \pm i \delta \\
\left(J_{0}^{-1}\right)_{i i}^{K} & =0 .
\end{aligned}
$$

Unlike the single channel case, the solution of these equations at finite voltage is complicated by the need to selfconsistently compute the distribution functions. This is because the hybridization no longer commutes with the Keldysh Green's functions of the conduction sea. In the following we limit our calculation to the linear response at zero voltage, which can be written in terms of the equilibrium $V=0$ Green's functions.

In equilibrium at $V=0$, the Green's functions are channel-diagonal

$$
\begin{aligned}
\hat{G}_{\psi \psi}^{-1} & =\hat{g}_{\psi \psi}^{-1}-\hat{\Sigma}_{\psi \psi}, \\
\hat{G}_{\varphi \varphi}^{-1} & =\hat{g}_{\varphi \varphi}^{-1}-\hat{\Sigma}_{\varphi \varphi}, \\
\hat{J}_{11}^{-1} & \left.=\hat{(} J_{11}^{0}\right)^{-1}-\hat{\Pi}_{11}, \\
\hat{J}_{22}^{-1} & \left.=\hat{(J}_{22}^{0}\right)^{-1}-\hat{\Pi}_{22} .
\end{aligned}
$$

(a)

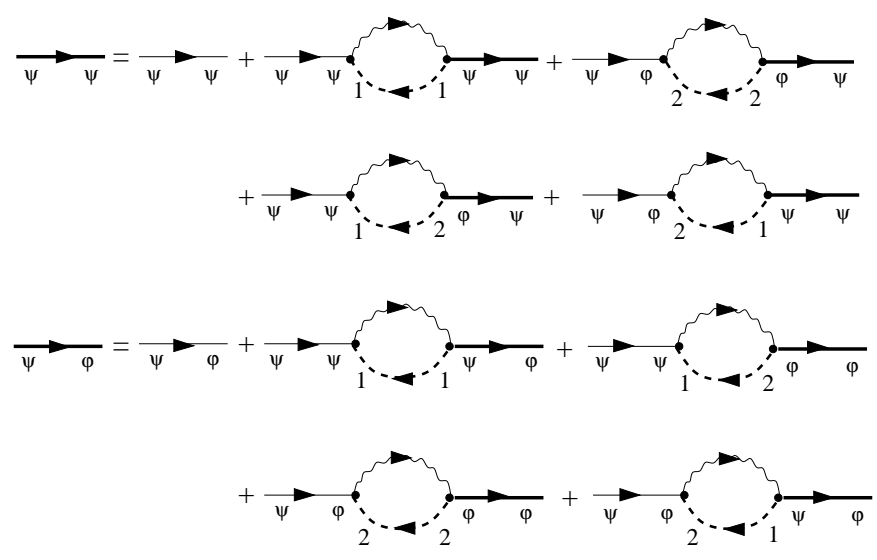

(b)

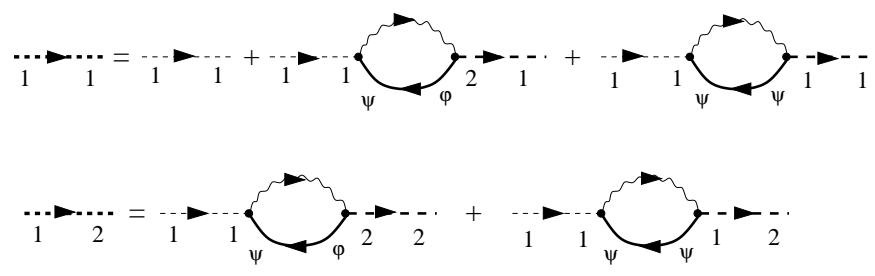

FIG. 6: Dyson equations for the conduction electron propagators (a) and the slave fermion propagators (b) for the twochannel quantum dot.

In this case the single-channel contributions to the current (56) can be expressed via "channel" t-matrices

$$
\begin{gathered}
I_{\psi}=\frac{2 e \rho}{\hbar}(\alpha \beta)^{2} \int d \omega\left(f_{L}(\omega)-f_{R}(\omega)\right) \operatorname{Im} T_{\psi}(\omega) \\
I_{\varphi}=\frac{2 e \rho}{\hbar}(\alpha \beta)^{2} \int d \omega\left(f_{L}(\omega)-f_{R}(\omega)\right) \operatorname{Im} T_{\varphi}(\omega),
\end{gathered}
$$

where $T_{\psi}=\Sigma_{\psi \psi}^{A}\left(1-i \pi \rho \Sigma_{\psi \psi}^{A}\right)^{-1}$, and $T_{\varphi}=\Sigma_{\varphi \varphi}^{A}(1-$ $\left.i \pi \rho \Sigma_{\varphi \varphi}^{A}\right)^{-1}$ are the advanced t-matrices. In linear response the single-channel t-matrices are proportional to the diagonal Green's function of the electrons on the dot holds a simple relation between channel Green's functions and the Green's functions of the physical electrons on the dot

$$
\begin{aligned}
& T_{\psi}(\omega)=\left(t_{1}\right)^{2} D_{11}^{A}(\omega), \\
& T_{\varphi}(\omega)=\left(t_{2}\right)^{2} D_{22}^{A}(\omega) .
\end{aligned}
$$

where

$$
D_{\lambda \lambda^{\prime}}^{A}(\omega)=i \int_{-\infty}^{0}\left\langle\left\{X_{\lambda \sigma}(t), X_{\lambda \sigma}^{\dagger}(0)\right\}\right\rangle e^{i \omega t} d t
$$

and $X_{\lambda \sigma}^{\dagger}=\frac{1}{\sqrt{2 S}} \sum_{\mu=1, K} b_{\sigma \mu}^{\dagger} \chi_{\mu}$. In the large $N$ limit, the t-matrices for each channel completely decouple in 
equilibrium, and are given by the scaling form

$$
T_{\lambda}(\omega, T)=\mathcal{T}\left(\frac{\omega}{T_{K \lambda}}, \frac{T}{T_{K \lambda}}\right)
$$

where $T_{K \lambda}(\lambda=1,2)$ are the Kondo temperatures in the two channels.

The interference contributions to the current pose a greater challenge and we have been unable to treat these terms without making some additional degree of approximation. Without loss of generality, the Keldysh components of the dot Green's functions can be written

$$
D^{K}=D^{R}\left(-D^{-1}\right)^{K} D^{A}
$$

In linear response, the effect of the voltage on will appear through the voltage dependence of $\left(D^{-1}\right)^{K}$, so we can replace the retarded and advanced combinations in the above by their channel diagonal components,

$$
\begin{aligned}
& D_{12}^{K}=D_{11}^{R}\left(-D^{-1}\right)_{12}^{K} D_{22}^{A} \\
& D_{21}^{K}=D_{22}^{R}\left(-D^{-1}\right)_{21}^{K} D_{11}^{A},
\end{aligned}
$$

In general, the Keldysh components of $D_{12}^{-1}$ and $D_{21}^{-1}$ contain a hybridization component and a term derived from interactions

$$
\begin{aligned}
& \left(-D_{12}^{-1}\right)^{K}=t_{1} g_{\psi \varphi}^{K} t_{2}-\left(t_{1} t_{2}\right) \Sigma_{12}^{K} \\
& \left(-D_{21}^{-1}\right)^{K}=t_{2} g_{\varphi \psi}^{K} t_{1}-\left(t_{1} t_{2}\right) \Sigma_{21}^{K}
\end{aligned}
$$

The second terms are a kind of vertex correction to the current operator (see Fig. 10 (a)). These terms describe the inelastic corrections to the interference contribution to the current. Our approximation entails neglecting these vertex corrections, so that (see Appendix III)

$$
\begin{aligned}
& D_{12}^{K}=t_{1} t_{2} D_{11}^{R} g_{\psi \varphi}^{K} D_{22}^{A} \\
& D_{21}^{K}=t_{1} t_{2} D_{22}^{R} g_{\varphi \psi}^{K} D_{11}^{A},
\end{aligned}
$$

When the same vertex corrections are ignored, the retarded and advanced Green's functions are channeldiagonal, $D_{12}^{R, A}=D_{21}^{R, A}=0$.

With these approximations, the contribution to the current due to the interference between the two channels becomes

$$
\begin{array}{r}
I_{\text {int }}=-\frac{2 e}{\hbar}\left(\alpha \beta t_{1} t_{2}\right)^{2} \pi \rho^{2} \int d \omega\left(f_{L}(\omega)-f_{R}(\omega)\right) \\
{\left[D_{11}^{R}(\omega) D_{22}^{A}(\omega)+D_{11}^{A}(\omega) D_{22}^{R}(\omega)\right],}
\end{array}
$$

or,

$$
\begin{array}{r}
I_{\text {int }}=-\frac{2 e}{\hbar}(\alpha \beta)^{2} \pi \rho^{2} \int d \omega\left(f_{L}(\omega)-f_{R}(\omega)\right) \\
{\left[T_{1}^{*}(\omega) T_{2}(\omega)+T_{1}(\omega) T_{2}^{*}(\omega)\right] .}
\end{array}
$$

This expression brings out the interference character of the term, and combined with (63) describes the linear response current flow, purely in terms of the channel diagonal, equilibrium t-matrics.
We can further simplify the full expression for the linear response current by taking advantage of the optical theorem. In the case where the scattering off the impurity is purely elastic, the channel diagonal t-matrices satisfy an optical theorem $\operatorname{Im} T_{\lambda}(\omega)=\pi \rho\left|T_{\lambda}(\omega)\right|^{2}$. The deviation from the optical theorem describes a decoherence scattering rate $\mathrm{en}^{23}$ as follows

$$
\tau_{\lambda}^{-1}=\operatorname{Im} T_{\lambda}(\omega)-\pi \rho\left|T_{\lambda}(\omega)\right|^{2} .
$$

In this form, the combined interference and channel diagonal currents can be written in the simple form

$$
G=G_{c o h}+G_{i n c}
$$

where

$$
G_{c o h}=\frac{N e^{2}}{h}(2 \alpha \beta)^{2} \int d \omega\left(-\frac{d f(\omega)}{d \omega}\right)\left|\pi \rho\left(T_{1}(\omega)-T_{2}(\omega)\right)\right|^{2}
$$

defines the coherent conductance through the twochannel and

$$
\begin{aligned}
G_{i n c} & =\frac{N e^{2}}{h}(2 \alpha \beta)^{2} \int d \omega\left(-\frac{d f(\omega)}{d \omega}\right) \\
& \times \pi \rho\left[\tau_{1}^{-1}(\omega)+\tau_{2}^{-1}(\omega)\right]
\end{aligned}
$$

the additional contribution due to incoherent transport. In the limits where the scattering t-matrix is purely elastic,

$$
T_{\lambda}(\omega)=\frac{e^{i \delta_{\lambda}(\omega)} \sin \delta_{\lambda}(\omega)}{\pi \rho}
$$

so that the inelastic scattering rate $\tau_{\lambda}^{-1}=0$ so at low temperatures

$$
\begin{aligned}
G_{c o h} & =\frac{N e^{2}}{h}(2 \alpha \beta)^{2}(\pi \rho)^{2}\left|T_{1}(0)-T_{2}(0)\right|^{2} \\
& =\frac{N e^{2}}{h}(2 \alpha \beta)^{2} \sin ^{2}\left(\delta_{1}-\delta_{2}\right)
\end{aligned}
$$

recovering the result expected from Landauer theory.

In the large $N$ limit it is straightforward to compute this approximate expression for the conductance. The scaling form (67) can be used to rescale the t-matrix from one channel to the other. Thus if $T_{K 1} / T_{K 2}=\alpha$, then from (67),

$$
T_{2}(\omega, T)=T_{1}(\alpha \omega, \alpha T) .
$$

The behavior of the linear conductance in case of the two-channel quantum dot for different ratios of the two Kondo temperatures is shown on Fig. 7. When the two Kondo temperatures are equal to each other, the conductance is completely suppressed due to destructive interchannel interference. When $T_{K 1}$ and $T_{K 2}$ are widely separated, at low temperatures conductance is suppressed as expected, and at high temperatures it develops a characteristic hump-structure due to the Kondo resonance in one of the channels. 


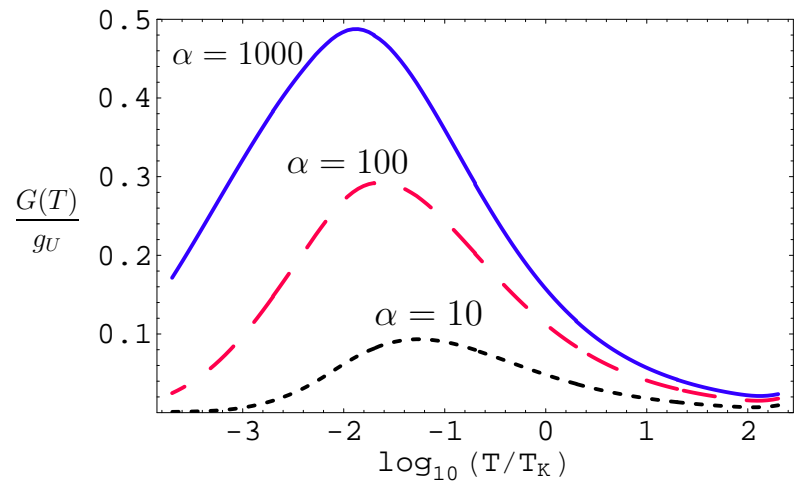

FIG. 7: Linear conductance of the two-channel quantum dot normalized with respect to $g_{U}=N \frac{e^{2}}{h} \sin ^{2}(\pi K / N)$, calculated for $K / N=0.4$ and $\alpha=\beta=1 / \sqrt{2}$, using equations (74) and (74), for three ratios of the two Kondo temperatures: $\alpha=$ $T_{K 1} / T_{K 2}=10$ (short-dashed curve), $\alpha=T_{K 1} / T_{K 2}=100$ (long-dashed curve) and $\alpha=T_{K 1} / T_{K 2}=1000$ (solid curve).

\section{CONCLUSION AND DISCUSSION.}

This article has considered the physics of a quantum dot in which Hund's coupling between the electrons localized within the dot gives rise to a spin-1 configuration on the quantum dot. Theoretically, such a quantum dot is expected to map onto a two-channel, spin-1 Kondo model in which a $\pi / 2$ phase shift develops in both scattering channels. According to a Landauer analysis of the resulting Fermi liquid, the transmission through the dot should vanish at low temperatures, giving rise to a conductance

$$
G=2 \frac{e^{2}}{h} \sin ^{2}\left(\delta_{1}-\delta_{2}\right)
$$

that vanishes at low temperatures $\frac{16,17}{}$. Paradoxically, experiment suggests that spin-1 quantum dots do indeed develop a zero-bias anomaly ${ }^{3,4,15}$. It is this issue that has motivated the current body of work.

Motivated by these results, the current authors proposed $^{21}$ that triplet quantum dots with a large zero bias correspond to anisotropic spin-1 Kondo models, in which the Kondo temperatures of the two channels exhibit a large ratio. In this paper, we have explored the general problem of high-spin quantum dots, screened by two conduction channels, attempting to develop a framework to model the detailed conductance of such systems. Our proposed model involves an infinite $U$ description of the electrons in the quantum dot, with an infinitely strong Hund's interaction that ensures the formation of a high-spin electron configuration in the dot. In this case the ground state of the dot is a spin-triplet and the charge fluctuations to the excited singly occupied states can be eliminated via Schrieffer-Wolff transformation. The Hamiltonian which describes the spin-1 quantum dot at low temperatures is then that of a two-channel Kondo Hamiltonian with two different Kondo coupling constants, and two different Kondo temperatures for the two electron states inside the triplet.

The emergence of two scattering channels in a spin-1 quantum dot has interesting consequences: in particular, the conductance develops a a non-monotonic dependence on magnetic field, temperature and voltage ${ }^{16}$. At zero temperature, destructive interference between the two elastic scattering channels leads to a complete suppression of conductance and the resulting ground state is described within a Landau Fermi liquid picture. Conductance starts gradually increasing with temperature and between $T_{K 2}$ and $T_{K 1}$ reaches it's maximum (the height of the maximum which is less or equal to the unitary conductance, depends on the difference between the two Kondo temperatures). At still higher temperatures $T>>T_{K 1}$, the conductance is ultimately suppressed to zero. Note that when the two Kondo temperatures are equal to each other, conductance remains suppressed for all temperatures 17 .

Our analytic analysis of this non-monotonic dependence of conductance on temperature employs a a Schwinger boson formalism to describe the dot spin degrees of freedom, using a large- $\mathrm{N}$ expansion to provide an approximate treatment of the resulting many body physics. Although the bosons in our approach remain unrenormalized, which means we neglect the spindecoherence effects we find that the that the initial increase, and ultimate suppression of conductance are reproduced in our analysis.

As part of this work, we considered the interesting case when one of the scattering channels is completely suppressed, which provides a realization of the underscreened Kondo effect in the spin-1 quantum dot. In this case at zero temperature conductance reaches its unitary limit, but in a specific singular way. Although the scattering shift at $T=0$ is equal to $\delta=\pi / 2$, the energy dependence of $\delta$ contains logarithmic contributions. This situation is described in terms of singular Fermi liquid 19 . It turns out that our Schwinger boson approximation is suited for description of a singular behavior of conductance at low temperatures and low voltages.

There are several open questions, of both a theoretical, and experimental nature that arise from this work. The current through the one-channel quantum dot is compactly related to the density of states of the dotelectrons via the Meir and Wingreen relation. In the two-channel case, these simplifications continue to operate in the contributions to the current that are channel diagonal, but the off-diagonal components to the current require a knowledge of the Keldysh Green's functions and the voltage-induced components to interchannel tmatrix. Is the physical reason for this added complexity? One possibility, that we have not been able to eliminate, is that a more general matrix ansatz for the Keldysh Green's functions, of the form, such as

$$
G^{K}(\omega)=G^{R}(\omega) F(\omega)-F(\omega) G^{A}(\omega)
$$

can be used to simplify our results. 
On the experimental front, we are still lacking hard evidence that the zero-bias anomaly in triplet dots is driven by an extreme ratio of Kondo temperatures. It would be particularly interesting if lateral quantum dots of the kind used in previous experiments could be adapted to support a variable ratio between the Kondo temperatures in the two channels. This might be done, for example, through the introduction of additional gates that change the symmetry of the quantum dot. This might provide the means to observe the cross-over from the singular Fermi liquid associated with fully developed underscreened behavior, to the interference dominated, nonmonotonic conductance of the fully screened quantum dot.

The authors wish to thank H. Kroha, G. Schon and G. Zarand for discussions and comments related to this work. We are particularly indebted to M. Eschrig for many useful discussions. This research was partly supported by DFG CFN (AP, BB) and DOE grant DEFG02-00ER45790 (PC).

\section{Appendix I: Self-energies for the one channel Kondo effect.}

The self-energy contributions to the equations (31) and (32) are shown in Fig.8. Each vertex is associated with a factor $\frac{i \tilde{t}}{\sqrt{N}}$. Internal summations over the spin index, or replica indices inside the loop provide a factor of $N$ or $K$ respectively, so that the conduction and slave fermion self energies are of order $O(N / N)=O(1)$ and $O(K / N)$ respectively, and they remain finite in the large $N$ limit. By contrast, the bosonic self-energy contains no internal summation over internal quantum numbers, so that this quantity is of order $O(1 / N)$ and vanishes in the large $N$ limit.

The conduction electron self energy is directly related to the Green's function of the d-electron, written in composite form as

$$
\Sigma_{ \pm \pm}\left(t-t^{\prime}\right)=\frac{-i \tilde{t}^{2}}{N} \sum_{\mu}\left\langle T_{c_{K}}\left(\chi^{\dagger} b_{\sigma \mu}\right)(t)\left(b_{\sigma \mu}^{\dagger} \chi^{\dagger}\right)\left(t^{\prime}\right)\right\rangle
$$

where $T_{c_{K}}$ refers to time-ordering along the Keldysh contour and the notation $A_{ \pm} \equiv A_{1} \pm A_{2}$ denotes the symmetric and antisymmetric combination of fields from the forwards time (1) and backwards time (2) parts of the contour. (Sometimes $A_{+} \equiv A_{c l}$ and $A_{-} \equiv A_{q}$ are referred to as the classical and quantum fields, respectively.)

The retarded, advanced and Keldysh combinations of the self energy are related to

$$
\Sigma_{R} \equiv \Sigma_{+-}, \quad \Sigma_{A} \equiv \Sigma_{-+}, \quad \Sigma_{K} \equiv \Sigma_{++} .
$$

The "classical" $(+)$ vertices have an off-diagonal structure in Kedlysh space, while the quantum (-) vertices are diagonal

$$
+\equiv \frac{1}{\sqrt{2}} \tau_{1}, \quad-\equiv \frac{1}{\sqrt{2}} \underline{1} .
$$

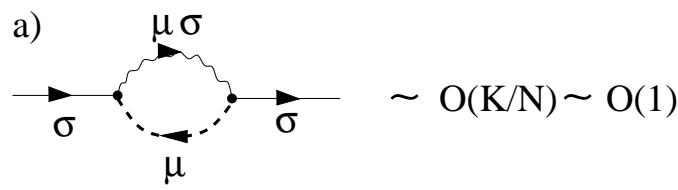

b)
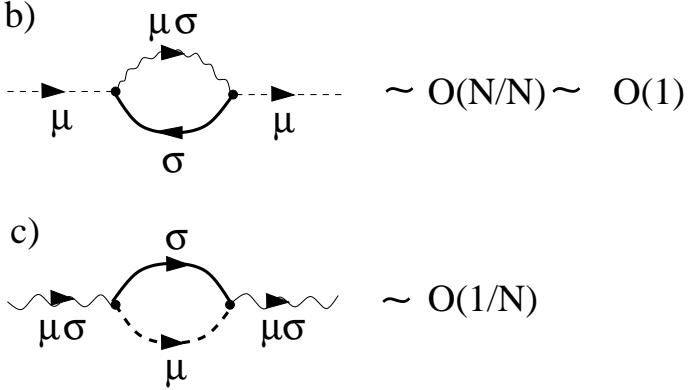

FIG. 8: NCA contributions to the self energies of a) lead electrons, b) auxiliary $\chi$-holes, and c) Schwinger bosons. The solid line denotes the Larkin Ovchinnikov matrix propagator for the conduction electrons. The dashed line denotes the corresponding Green's function of the auxiliary $(\chi)$ fermions and the wavy line is the bosonic propagator. Each vertex corresponds to the factor $i \frac{\tilde{t}}{\sqrt{N}}$.

With this information, the conduction electron self energy is given by

$$
\Sigma(t)=\left[\begin{array}{cc}
\Sigma_{R}(t) & \Sigma_{K}(t) \\
& \Sigma_{A}(t)
\end{array}\right] \equiv\left[\begin{array}{cc}
\Sigma_{+-}(t) & \Sigma_{++}(t) \\
0 & \Sigma_{-+}(t)
\end{array}\right]
$$

which in expanded form gives

$$
\Sigma(t)=i \frac{K}{2 N}\left[\begin{array}{cc}
\operatorname{Tr}\left[\tau_{1} B(t) J(-t)\right] & \operatorname{Tr}\left[\tau_{1} B(t) \tau_{1} J(-t)\right] \\
0 & \operatorname{Tr}\left[B(t) \tau_{1} J(-t)\right]
\end{array}\right]
$$

The Keldysh traces give the combinations

$$
\begin{array}{r}
\operatorname{Tr}\left[\tau_{1} B J\right] \equiv B_{R} J_{K}+B_{K} J_{A} \\
\operatorname{Tr}\left[\tau_{1} B \tau_{1} J\right] \equiv B_{R} J_{A}+B_{A} J_{R}+B_{K} J_{K} \\
\operatorname{Tr}\left[B \tau_{1} J\right] \equiv B_{K} J_{A}+B_{R} J_{K}
\end{array}
$$

Carrying out the Fourier transforms, the explicit form for the conduction self-energy is then

$$
\begin{aligned}
\Sigma^{R}(\epsilon) & =-i t^{2} \frac{K}{N} \int \frac{d \omega}{4 \pi}\left[B^{R}(\omega) J^{K}(\omega-\epsilon)\right. \\
& \left.+B^{K}(\omega) J^{A}(\omega-\epsilon)\right] \\
\Sigma^{A}(\epsilon) & =-i t^{2} \frac{K}{N} \int \frac{d \omega}{4 \pi}\left[B^{K}(\omega) J^{R}(\omega-\epsilon)\right. \\
& \left.+B^{A}(\omega) J^{K}(\omega-\epsilon)\right] \\
\Sigma^{K}(\epsilon) & =-i t^{2} \frac{K}{N} \int \frac{d \omega}{4 \pi}\left[B^{K}(\omega) J^{K}(\omega-\epsilon)\right. \\
& \left.+B^{A}(\omega) J^{R}(\omega-\epsilon)+B^{R}(\omega) J^{A}(\omega-\epsilon)\right]
\end{aligned}
$$

Repeating the same procedure for the slave fermions, we 
obtain

$$
\begin{aligned}
\Pi^{R}(\epsilon) & =-i t^{2} \int \frac{d \omega}{4 \pi}\left[B^{R}(\omega) G^{K}(\omega-\epsilon)\right. \\
& \left.+B^{K}(\omega) G^{A}(\omega-\epsilon)\right] \\
\Pi^{A}(\epsilon) & =-i t^{2} \int \frac{d \omega}{4 \pi}\left[B^{K}(\omega) G^{R}(\omega-\epsilon)\right. \\
& \left.+B^{A}(\omega) G^{K}(\omega-\epsilon)\right] \\
\Pi^{K}(\epsilon) & =-i t^{2} \int \frac{d \omega}{4 \pi}\left[B^{K}(\omega) G^{K}(\omega-\epsilon)\right. \\
& \left.+B^{A}(\omega) G^{R}(\omega-\epsilon)+B^{R}(\omega) G^{A}(\omega-\epsilon)\right]
\end{aligned}
$$

Since the Schwinger boson self-energy vanishes in the large $N$ limit, the Schwinger boson fields are unrenormalized and take their bare values

$$
\begin{aligned}
B^{R, A}(\nu) & =\frac{1}{\nu-\lambda \pm i \delta} \\
B^{K}(\nu) & =-2 \pi i \delta(\nu-\lambda) h_{B}
\end{aligned}
$$

where $h_{B}=1+2 n_{B}$ and $n_{B} \equiv n_{B}(\lambda)=2 S K /(N K)$ is the Bose distribution function. The Keldysh Green's function of the fermions can be related to their retarded and advanced Green functions via the relations

$$
\begin{aligned}
J^{K}(\omega) & =\left(J^{R}(\omega)-J^{A}(\omega)\right) h_{\chi}(\omega) \\
& =2 i \operatorname{Im}\left[J_{R}(\omega)\right]\left(1-2 f_{\chi}(\omega)\right), \\
G^{K}(\omega) & =\left(G^{R}(\omega)-G^{A}(\omega)\right) h_{\Psi}(\omega) \\
& =2 i \operatorname{Im}\left[G_{R}(\omega)\right]\left(1-2 f_{\Psi}(\omega)\right) .
\end{aligned}
$$

With these simplifications, we can expand the selfenergies in the following form

$$
\begin{aligned}
\Pi^{A}(\epsilon) & =-t^{2} n_{B}(\lambda) G^{R}(\lambda-\epsilon) \\
& -t^{2} \int_{-\infty}^{\infty} \frac{d \omega}{\pi} f_{\Psi}(\omega) \frac{1}{\omega-\lambda+\epsilon-i \delta} \operatorname{Im}^{R}(\omega) \\
\Pi^{R}(\epsilon) & =-t^{2} n_{B}(\lambda) G^{A}(\lambda-\epsilon) \\
& -t^{2} \int_{-\infty}^{\infty} \frac{d \omega}{\pi} f_{\Psi}(\omega) \frac{1}{\omega-\lambda+\epsilon+i \delta} \operatorname{Im} G^{R}(\omega) \\
\Pi^{K}(\epsilon) & =-2 i t^{2} \operatorname{Im} G^{R}(\lambda-\epsilon)\left[n_{B}(\lambda)-f_{\Psi}(\lambda-\epsilon)\right. \\
& \left.-2 n_{B}(\lambda) f_{\Psi}(\lambda-\epsilon)\right]
\end{aligned}
$$

The slave fermion distribution function is given by

$$
h_{\chi}=\frac{\Pi_{\chi}^{K}}{\Pi_{\chi}^{R}-\Pi_{\chi}^{A}}=\frac{h_{\Psi} h_{B}-1}{h_{B}-h_{\Psi}} .
$$

Rearranging this expression gives

$$
h_{\psi}(\omega)=\frac{h_{\chi} h_{B}-1}{h_{B}-h_{\chi}} .
$$

Similarly,

$$
\begin{aligned}
& \Sigma^{A}(\epsilon)=-t^{2} \frac{K}{N} n_{B}(\lambda) J^{R}(\lambda-\epsilon) \\
& -t^{2} \frac{K}{N} \int_{-\infty}^{\infty} \frac{d \omega}{\pi} f_{\chi}(\omega) \frac{1}{\omega-\lambda+\epsilon-i \delta} \operatorname{Im} J^{R}(\omega) \\
& \Sigma^{R}(\epsilon)=-t^{2} \frac{K}{N} n_{B}(\lambda) J^{A}(\lambda-\epsilon) \\
& -t^{2} \frac{K}{N} \int_{-\infty}^{\infty} \frac{d \omega}{\pi} f_{\chi}(\omega) \frac{1}{\omega-\lambda+\epsilon+i \delta} \operatorname{Im}^{R}(\omega) \\
& \Sigma^{K}(\epsilon)=-2 i t^{2} \frac{K}{N} \operatorname{Im} J^{R}(\lambda-\epsilon)\left[n_{B}(\lambda)-f_{\chi}(\lambda-\epsilon)\right. \\
& \text { - } \left.2 n_{B}(\lambda) f_{\chi}(\lambda-\epsilon)\right]
\end{aligned}
$$

We can also compute the electron distribution function from

$$
h_{\psi}(\omega)=\frac{\Sigma^{K}}{\Sigma_{\chi}^{R}-\Sigma_{\chi}^{A}}=\frac{h_{\chi} h_{B}-1}{h_{B}-h_{\chi}} .
$$

but this recovers exactly the result obtained in (86), showing that detailed balance is satisfied.

\section{Appendix II: on the derivation of general current formula in case of two channels}

As an example we present the derivation of the Green's function $G_{1 \varphi}$

$$
G_{1 \varphi}\left(t_{1}, t_{2}\right)=-i\left\langle T_{c k} d_{1}\left(t_{1}\right) \varphi^{+}\left(t_{2}\right)\right\rangle,
$$

and the Dyson Eq is that depicted on Fig.4 To avoid dou-

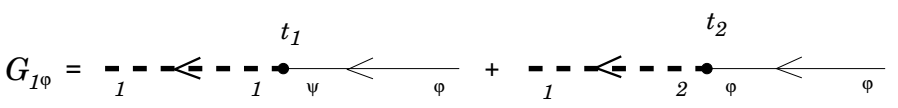

FIG. 9: The Dyson Eq. for $\hat{G}_{1 \varphi}$ Green's function $\left(\hat{G}_{1 \varphi}\right.$ is a matrix in Keldysh space).

ble counting we renormalize only the $d$-electron Green's function, while the channel Green's function is left unrenormalized, so that the expression for $G_{1 a}$ is

$$
\begin{aligned}
G_{1 \varphi}\left(t_{1}, t_{2}\right)=\int_{c K} d & \tau\left(\hat{D}_{11}\left(t_{1}, \tau\right) t_{1} \hat{g}_{\psi \varphi}\left(\tau, t_{2}\right)\right. \\
& \left.+\hat{D}_{12}\left(t_{1}, \tau\right) t_{2} \hat{g}_{\varphi \varphi}\left(\tau, t_{2}\right)\right)
\end{aligned}
$$

where

$$
D_{i j}\left(t_{1}, t_{2}\right)=-i\left\langle T_{c k} d_{i}\left(t_{1}\right) d_{j}^{+}\left(t_{2}\right)\right\rangle .
$$

Expression (90) can be rewritten in Larkin-Ovchinnikov space and the Keldysh part of the Green's function can be found. After Fourier transforming, we obtain the following Keldysh component

$$
G_{1 \varphi}^{K}=t_{1}\left(D_{11}^{R} g_{\psi \varphi}^{K}+D_{11}^{K} g_{\psi \varphi}^{A}\right)+t_{2}\left(D_{12}^{R} g_{\varphi \varphi}^{K}+D_{12}^{K} g_{\varphi \varphi}^{A}\right)
$$


The Dyson Eq for $G_{2 \psi}$ is derived in an analogous fashion

$$
G_{2 \psi}^{K}=t_{2}\left(D_{22}^{R} g_{\varphi \psi}^{K}+D_{22}^{K} g_{\varphi \psi}^{A}\right)+t_{1}\left(D_{21}^{R} g_{\psi \psi}^{K}+D_{21}^{K} g_{\psi \psi}^{A}\right)
$$

These equations can now be expanded using the explicit expressions for the bare channel Green's functions given in section IV,B to obtain the general expressions (56) and (57).

\section{Appendix III: estimation of the interference current term}

a)

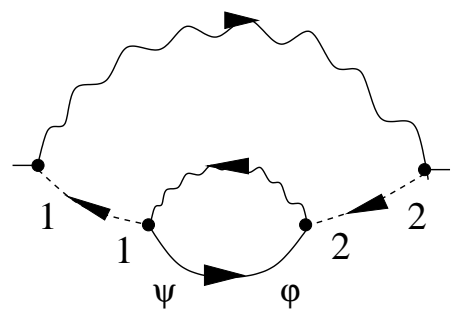

b)

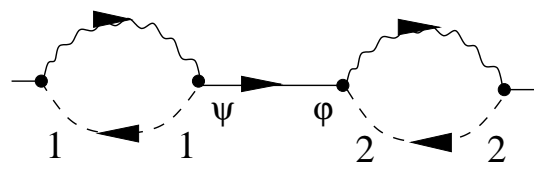

FIG. 10: Diagrammatic contributions to the off-diagonal Green's function $D_{12}(\omega)$, describing inter-channel contributions to the conduction electron propagator.

Contributions to the current which are produced by channel interference are defined by the interchannel Green's functions $D_{12}$ and $D_{21}$. These Green's functions are proportional to the self-energies of $G$-functions. On Fig. 10 we show two types of contributions which arise in the first order of the perturbation theory. Diagrams of type a) contribute to the non-elastic scattering of delectrons, and therefore constitute the non-Fermi liquid corrections to the conductance. Other diagrams can be summed in a simple Dyson equation

$$
\begin{aligned}
D_{12}=D_{11}^{0} t_{1} G_{\psi 2} & =D_{11}^{0} t_{1} g_{\psi \psi} t_{1} D_{12}+D_{11}^{0} t_{1} g_{\psi \varphi} t_{2} D_{22} \\
D_{12} & =t_{1} t_{2}\left(1-t_{1}^{2} D_{11}^{0} g_{\psi \psi}\right)^{-1} D_{11}^{0} g_{\psi \varphi} D_{22}(29)
\end{aligned}
$$

where, as usual $D_{11}^{0}$ are bare Green's functions, $g_{\psi \psi}, g_{\psi \varphi^{-}}$ bare conduction electron Green's functions, and $D_{12}, D_{22}$ are the fully-renormalized d-electron Green's functions.

Analogously we can write the Dyson Eq for $D_{11}$

$$
\begin{aligned}
D_{11} & =D_{11}^{0}+t_{1}^{2} D_{11}^{0} g_{\psi \psi} D_{11}+t_{1} t_{2} D_{11}^{0} g_{\psi \varphi} D_{21} \\
& =D_{11}^{0}+t_{1}^{2} D_{11} g_{\psi \psi} D_{11}^{0}+t_{1} t_{2} D_{12} g_{\varphi \psi} D_{11}^{0}
\end{aligned}
$$

We can write from here

$$
D_{11}\left(1-t_{1}^{2} g_{\psi \psi} D_{11}^{0}\right)=D_{11}^{0}\left(1+t_{1} t_{2} D_{12} g_{\varphi \psi}\right),
$$

so that

$$
D_{11}=\left(1+t_{1} t_{2} D_{12} g_{\varphi \psi}\right) D_{11}^{0}\left(1-t_{1}^{2} g_{\psi \psi} D_{11}^{0}\right)^{-1}
$$

and

$$
\begin{aligned}
\left(1+t_{1} t_{2} D_{12} g_{\varphi \psi}\right)^{-1} D_{11} & =D_{11}^{0}\left(1-t_{1}^{2} g_{\psi \psi} D_{11}^{0}\right)^{-1} \\
& =\left(1-t_{1}^{2} D_{11}^{0} g_{\psi \psi}\right)^{-1} D_{11}^{0}
\end{aligned}
$$

Now we can plug (98) into (94) and get

$$
D_{12}=\left(1+t_{1} t_{2} D_{12} g_{\varphi \psi}\right)^{-1} t_{1} t_{2} D_{11} g_{\psi \varphi} D_{22}
$$

which leads to the equation

$$
D_{12}=t_{1} t_{2} D_{11} g_{\psi \varphi} D_{22}-t_{1} t_{2} D_{12} g_{\varphi \psi} D_{12}
$$

From (100) we now can derive that retarded and advanced off-diagonal terms vanish in this approximation. Namely, for example

$$
D_{12}^{R}=t_{1} t_{2} D_{11}^{R} g_{\psi \varphi}^{R} D_{22}^{R}-t_{1} t_{2} D_{12}^{R} g_{\varphi \psi}^{R} D_{12}^{R}
$$

is correct and therefore $D_{12}^{R}=0$, because $g_{\psi \varphi}^{R}=g_{\varphi \psi}^{R}=0$. Keldysh components of $D_{12}$ nevertheless do not vanish

$$
\begin{aligned}
D_{12}^{K} & =t_{1} t_{2}\left(D_{11}^{R} g_{\psi \varphi}^{R} D_{22}^{K}+D_{11}^{R} g_{\psi \varphi}^{K} D_{22}^{A}+D_{11}^{K} g_{\psi \varphi}^{A} D_{22}^{A}\right)+ \\
& \left.+t_{1} t_{2}\left(D_{12}^{R} g_{\varphi \psi}^{R} D_{12}^{K}+D_{12}^{R} g_{\varphi \psi}^{K} D_{12}^{A}+D_{12}^{K} g_{\varphi \psi}^{A} D_{12}^{A}{ }_{1}^{A}\right) 02\right)
\end{aligned}
$$

Most of the terms in (102) vanish and we are left with

$$
D_{12}^{K}=t_{1} t_{2} D_{11}^{R} g_{\psi \varphi}^{K} D_{22}^{A}
$$

* Email: anna@tfp.physik.uni-karlsruhe.de

1 A. C. Hewson, The Kondo Problem to Heavy Fermions, Cambridge University Press, Cambridge, 1993.

${ }^{2}$ L. Kouwenhoven and L. Glazman, Physics World 14, 33 (2001).
${ }^{3}$ S. Sasaki, S. De Franceschi, J. M. Elzerman, W. G. van der Wiel, M. Eto, S. Tarucha, and L. P. Kouwenhoven, Nature 405, 764 (2000).

4 A. Kogan, G. Granger, M. A. Kastner, and D. GoldhaberGordon, Phys. Rev. B 67, 113309 (2003). 
5 Ph. Nozières and A. Blandin, J. Physique 41, 193 (1980).

6 N. Andrei and C. Destri, Phys. Rev. Lett. 52, 364 (1984).

7 I. Affleck, A. W. W. Ludwig, Phys. Rev. Lett. 67, 161 (1991).

8 D. L. Cox, Phys. Rev. Lett. 59, 1240 (1987).

${ }^{9}$ N. Andrei and C. J. Bolech, in "Concepts in Electron Correlation", edited by A. C. Hewson and V. Zlatic, NATO Science Series II: Mathematics, Physics and Chemistry Vol. 110 (Kluwer), (2003)

10 L. I. Glazman and M. E. Raikh, JETP Lett. 47, 452 (1988).

11 Yval Oreg and David Goldhaber-Gordon, Phys. Rev. Lett. 90, 136602 (2003).

12 Ron M. Potok, Ileana G. Rau, Hadas Shtrikman, Y. Ored, and David Goldhaber-Gordon, unpublished.

${ }^{13}$ D. Goldhaber-Gordon, H. Shtrikman, D. Mhalu, D. Abusch-Magder, U. Meirav, and M. A. Kastner, Nature 391, 156 (1998).
14 S. M. Cronenwett et al., Science 281, 540 (1998).

15 J. Schmid, J. Weis, K. Eberl, and K. v. Klitzing, Phys. Rev. Lett. 84, 5824 (2000).

16 M. Pustilnik and L. I. Glazman, Phys. Rev. Lett. 87, 216601 (2001).

17 W. Hofstetter and G. Zarand, Phys. Rev. B 69, 235301 (2004).

18 P. Coleman and C. Pepin, Phys. Rev. B 68, 220405 (2003).

19 P. Mehta, L. Borda, G.Zarand, N. Andrei, and P. Coleman, Phys. Rev. B 72, 014430 (2005).

20 P. Coleman and I. Paul, Phys. Rev. B 71, 035111 (2005).

21 A. Posazhennikova and P. Coleman, Phys. Rev. Lett. 94, 036802 (2005).

${ }^{22}$ Y. Meir, N. S. Wingreen, Phys. Rev. Lett. 68, 2512 (1992).

${ }^{23}$ G. Zarand, L. Borda, J. von Delft and N.Andrei Phys. Rev. Lett. 93, 107204 (2004) 